\title{
Spin Forms and Spin Interactions among Higgs Bosons, between Higgs Boson and Graviton
}

\author{
ShaoXu Ren \\ Institute of Physical Science and Engineering \\ Tongji University, 200092, Shanghai, China \\ Corresponding email: shaoxu-ren@hotmail.com
}

Received 16 March 2016; accepted 25 April 2016; published 29 April 2016

\section{Abstract}

This paper offers concrete spin matrix forms of $0 h$ spin zero particle, and shows the existent of the spin interactions among $0 \hbar$ spin zero particles. It is obviously hoping to approach, on the most comprehensive level, to understand what really Higgs Boson is and what role-play Higgs Boson is acting in particle physics.

As a "particle" of gravitational force, the spin interaction between $0 \hbar$ spin zero particle (Higgs Boson) and $2 \hbar$ spin particle (Graviton) is given, which maybe a way that people would find Graviton in future.

\section{Keywords}

Higgs Boson; Graviton; Vacuum Bubble Pair; Spin Topological Space, STS ; Casimir operator; right-hand $0 \hbar$ spin zero particle; left-hand $0 h$ spin zero particle

\section{Introduction}

Higgs particle is a fundamental particle predicted by the Standard Model, and confirmed by the Large Hadron Collider at CERN.[1] Higgs particle could explain why the photon has no mass while $W$ and $Z$ Bosons are very heavy in electron-weak theory, and endows Fermions such as electron, muon and tau particle and quarks with their masses. The intrinsic spin angular momentum of Higgs particle is $0 \hbar$. In conventional quantum mechanics, the representation of spin zero particle is a zero-matrix of one dimensional space, this means, in fact, Higgs particle has no matrix representation for its spin property in the Standard Model. What a pity ! magical Higgs particle could create masses of the particles in universe, however, failing to write out its own non-trivial spin matrices. Zero is not nothing, zero spin is not non-twirling. From the pointview of The Third Kind of Particles, TKP, [2] the angular momentum property of spin zero particle can be expressed by infinite dimensional non-Hermitian matrices which related to Vacuum Bubble Pair, VBP, 
these pairs could be excited into $0 h$ spin particle form phase transitions of Vacuum Spin Particle, VSP, whose Casimir Operator is $-\frac{1}{4} \hbar^{2} I_{0}$, less than zero. [3] ( Casimir Operator of Higgs particle is $0 \hbar^{2} I_{0}$, of Graviton is $6 \hbar^{2} I_{0}$ )

In conventional quantum mechanics, each particle has its own spin space: one spin prticle, one spin space; two spin particles, two spin spaces; ......; $n$ spin particles, $n$ spin spaces. These spin spaces are independent each other, and expressed by $V=V_{1} \otimes V_{2} \otimes V_{3} \otimes \ldots \otimes V_{\mathrm{n}}$.

This paper gives advice: Since the spin angular momentum constituents of every elementary particle are composed of the common series of math elements that based on the raising operators $\pi_{j}^{+}=\pi_{1 ; j, k}^{+}+i \pi_{2 ; j, k}^{-}$and lowering operators $\pi_{k}^{-}=\pi_{1 ; j, k}^{+}-i \pi_{2 ; j, k}^{-}$, (which compose VBP, TKP ), then a new type of spin space, the so-called Spin Topological Space, STS , [4] is established. All sorts of spin sparticles are attributed to this spin space, STS.

In traditional views, there are no any spin interactions among spin zero particles. However, by means of STS concept, on the contrary, it is shown there are the spin interactions. $\quad 0 h$ Spin zero particle not only possesses spin phenomena but also appears out right-circumrotation and left-circumrotation, such kind of properties may exist in Higgs Boson world of the Standard Model.

Same reason for, there should be spin particle interactions between Higgs Boson and graviton, and spin interactions among gravitons, detecting gravitational force, after the interference effect of gravitational wave is comfirmed. [5]

2. Higgs Boson's Spin angular Momentum matrices $\pi_{1}(l), \pi_{2}(l), \pi_{3}(l)$

The mathematical structure of $\pi_{1}(l), \pi_{2}(l), \pi_{3}(l)$ and $\pi_{3}^{2}(l), \pi_{1}^{2}(l)+\pi_{2}^{2}(l)$ are given in matrix series (1), (2), (3) shown below.

They satisfy angular momentum commutation rules

$$
\begin{aligned}
\pi_{i}(l) \pi_{j}(l)-\pi_{j}(l) \pi_{i}(l) & =i \pi_{k}(l) \\
i, j, k & =1,2,3 \text { are circulative }
\end{aligned}
$$

Casimir Operator

$$
\pi^{2}(l)=\pi_{1}^{2}(l)+\pi_{2}^{2}(l)+\pi_{3}^{2}(l)=0 I_{0}=0(0+1) I_{0}
$$

Using raising operator, lowering operator

$$
\pi^{+}(l)=\pi_{1}(l)+i \pi_{2}(l) \quad ; \quad \pi^{-}(l)=\pi_{1}(l)-i \pi_{2}(l)
$$

Then (0.1) turns to

$$
\begin{aligned}
& \pi_{3}(l) \pi^{+}(l)-\pi^{+}(l) \pi_{3}(l)=+\pi^{+}(l) \\
& \pi_{3}(l) \pi^{-}(l)-\pi^{-}(l) \pi_{3}(l)=-\pi^{-}(l) \\
& \pi^{+}(l) \pi^{-}(l)-\pi^{-}(l) \pi^{+}(l)=2 \pi_{3}(l)
\end{aligned}
$$

What follows are the explicit spin matrix representations of three generations $(l=1,2,3)$ of $0 \hbar$ Boson, (Higgs Boson). 


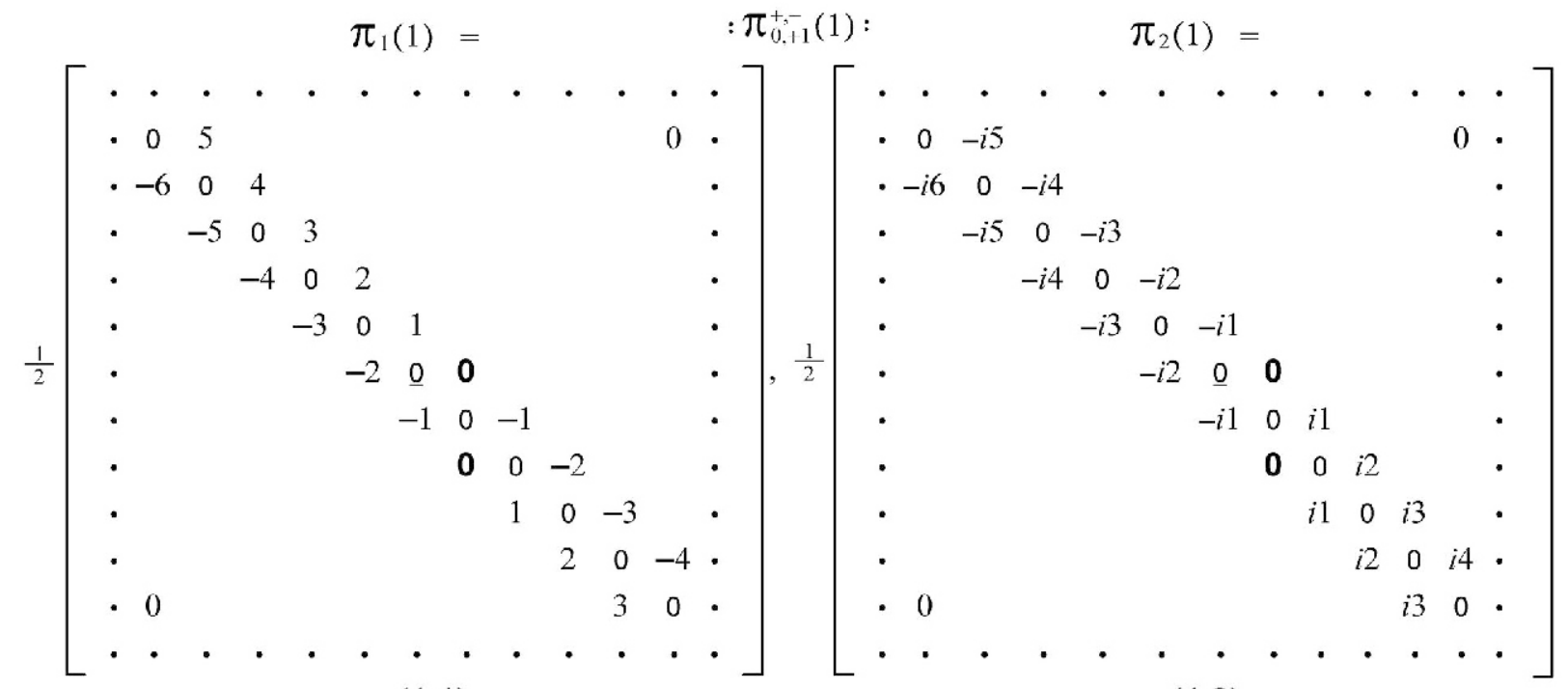

(1.1)

(1.2)
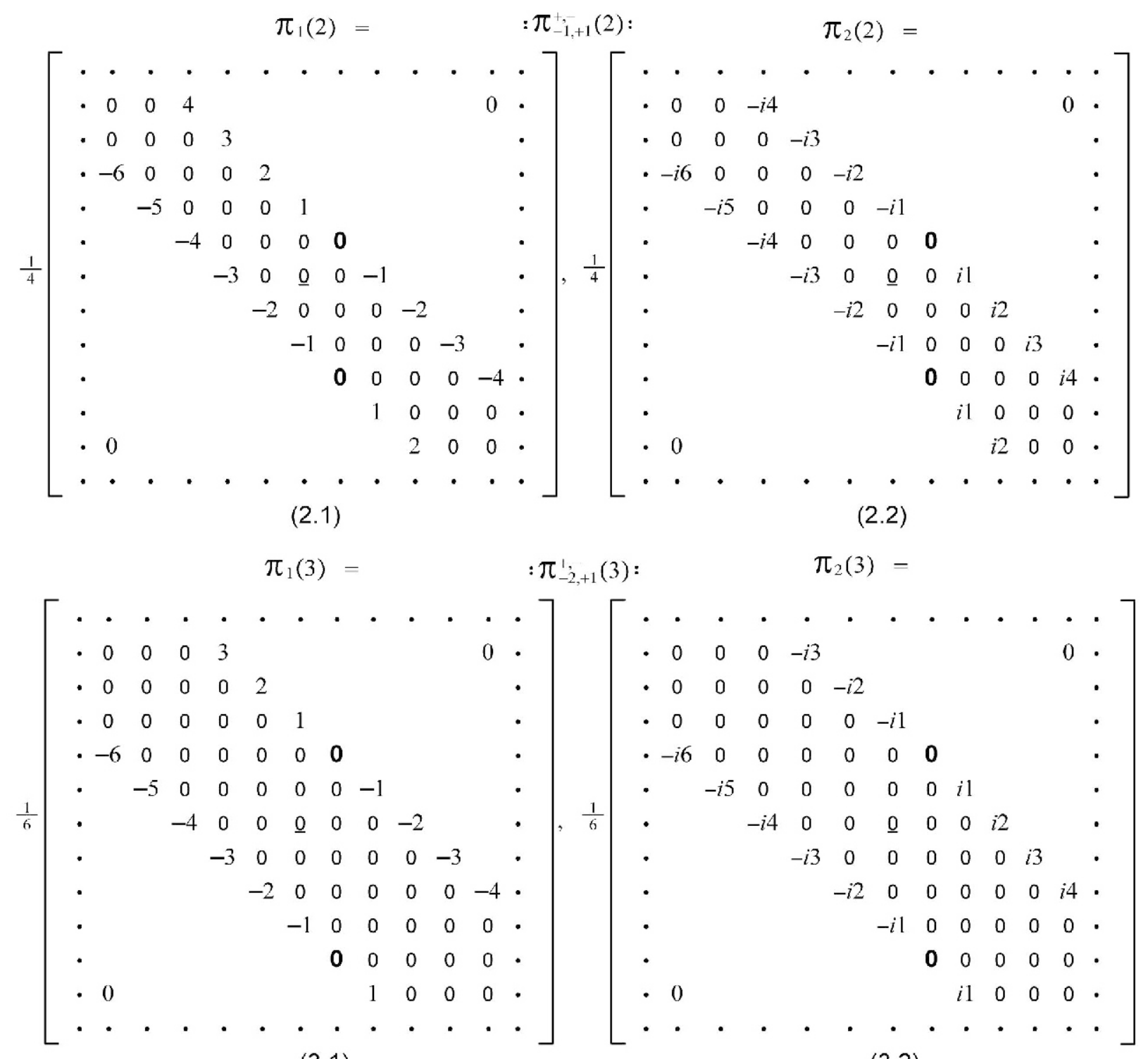

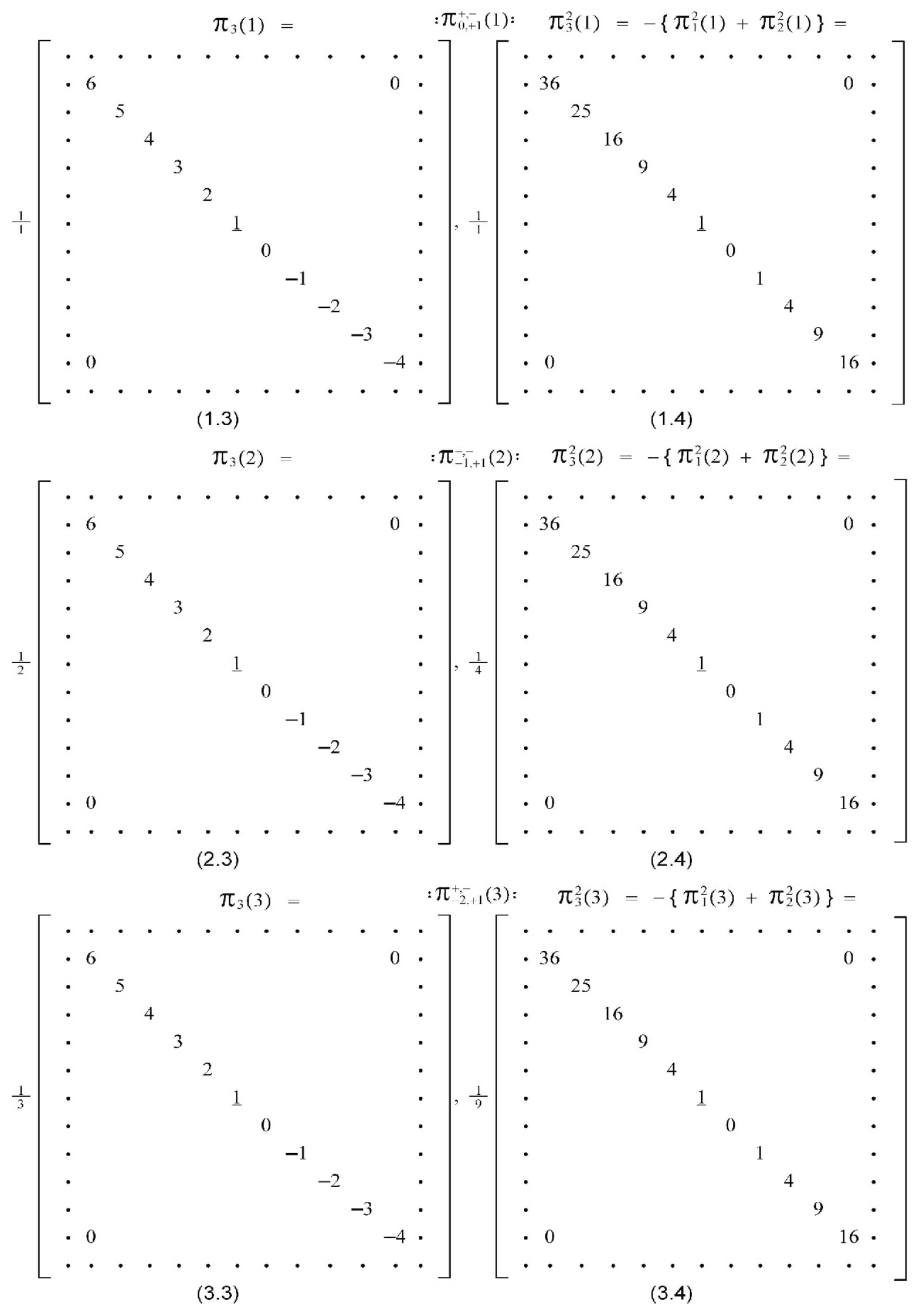

(3.3) 
Be brief, in Spin Topological Space, STS , [4], the above spin matrices \{ (1.1), (1.2), (1.3); (2.1), (2.2), (2.3); (3.1), (3.2), (3.3) \} of Higgs Boson can be rewritten in the spin forms of (4.1), (4.2), (4.3)

$$
\begin{aligned}
& \vec{\pi}_{0,+1}(1)=\left\{\pi_{1}(1), \pi_{2}(1), \pi_{3}(1)\right\} \\
& \vec{\pi}_{-1,+1}(2)=\left\{\pi_{1}(2), \pi_{2}(2), \pi_{3}(2)\right\} \\
& \vec{\pi}_{-2,+1}(3)=\left\{\pi_{1}(3), \pi_{2}(3), \pi_{3}(3)\right\}
\end{aligned}
$$

For an example of $\vec{\pi}_{0,+1}(1)$, now, (4.1) is denoted by (5.0):

$$
\begin{aligned}
\vec{\pi}_{0,+1}(1) & =\left\{\pi_{1 ; 0,+1}(1), \pi_{2 ; 0,+1}(1), \pi_{3 ; 0,+1}(1)\right\} \\
\pi_{1 ; 0,+1}(1) & =\frac{1}{2}\left(\pi_{0}^{+}+\pi_{+1}^{-}\right)=\pi_{1}(1) \\
\pi_{2 ; 0,+1}(1) & =\frac{1}{2 \mathrm{i}}\left(\pi_{0}^{+}-\pi_{+1}^{-}\right)=\pi_{2}(1) \\
\pi_{3 ; 0,+1}(1) & =\frac{1}{\mathrm{i}}\left\{\pi_{1 ; 0,+1}(1) \pi_{2 ; 0,+1}(1)-\pi_{2 ; 0,+1}(1) \pi_{1 ; 0,+1}(1)\right\}=\pi_{3}(1)
\end{aligned}
$$

\section{Spin Interactions between Two Higgs Bosons}

a) First we deal with two-body system that compose of Higgs Boson a, $\vec{\pi}_{a}$ and Higgs Boson b, $\vec{\pi}_{b} . \quad \vec{\pi}_{a}$ and $\vec{\pi}_{b}$ are their spin angular momentum matrix operators. Then show a case of a spin coupling interaction (6.0) between $\vec{\pi}_{a}$ and $\vec{\pi}_{b}$, the scalar products $\mathbb{S}^{2}(l)$, or Casimir operators of their three generations as follows

$$
\begin{aligned}
\mathbb{S}^{2}(l) & =\overrightarrow{\mathbb{S}}(l) \cdot \overrightarrow{\mathbb{S}}(l) \\
\text { Where } \quad \overrightarrow{\mathbb{S}}(l) & =\vec{\pi}_{a}(l)+\vec{\pi}_{b}(l) \\
\text { Or } \quad \overrightarrow{\mathbb{S}}_{0,+1 ;-2,-1}(1) & =\vec{\pi}_{0,+1}(1)+\vec{\pi}_{-2,-1}(1) \\
\overrightarrow{\mathbb{S}}_{-1,+1 ;-3,-1}(2) & =\vec{\pi}_{-1,+1}(2)+\vec{\pi}_{-3,-1}(2) \\
\overrightarrow{\mathbb{S}}_{-2,+1 ;-4,-1}(3) & =\vec{\pi}_{-2,+1}(3)+\vec{\pi}_{-4,-1}(3)
\end{aligned}
$$

After careful calculation, for $\mathbb{S}^{2}(6.0)$, we have (8.0)

$$
\text { Or } \begin{aligned}
\mathbb{S}^{2}(l) & =\overrightarrow{\mathbb{S}}(l) \cdot \overrightarrow{\mathbb{S}}(l)=0 I_{0} \\
\mathbb{S}_{0,+1 ;-2,-1}^{2}(1) & =0 I_{0} \\
\mathbb{S}_{-1,+1 ;-3,-1}^{2}(2) & =0 I_{0} \\
\mathbb{S}_{-2,+1 ;-4,-1}^{2}(3) & =0 I_{0} \\
I_{0} & =\operatorname{diag}\{\ldots, 1,1,1,1,1, \underline{1}, 1,1,1,1,1, \ldots\}(9.0)
\end{aligned}
$$

(8.0) and (8.1), (8.2), (8.3) show: there are no any effects of spin coupling bwtween two $0 h$ zero spin particles, $\vec{\pi}_{a}(l)$ and $\vec{\pi}_{b}(l)$. 
b) How can we find no-trivial spin-coupling interaction rather than (8), by using augular momentum operators of $0 \hbar$ zero spin particle ?

Actually there are two types of $0 \hbar$ zero spin particles, which is a way to overcome the obstacle

Assume $0 \hbar$ zero spin particles $\vec{\pi}_{0,+1}(1)$ and $\vec{\pi}_{-2,-1}(1)$ to be thought of as two right-hand spin particles, frmula (7.1) could be written as (10).

$$
\overrightarrow{A_{R}}=\overrightarrow{\mathbb{S}}_{0,+1 ;-2,-1}(1)=\vec{\pi}_{0,+1}(1)+\vec{\pi}_{-2,-1}(1)
$$

Further the formula (8.1) is expressed as (11)

$$
\overrightarrow{A_{R}} I_{0} \overrightarrow{A_{R}}=\mathbb{S}_{0,+1 ;-2,-1}^{2}(1)=0 I_{0}
$$

On the other side, ${\overrightarrow{A_{L}}}^{\circ}$ is marked as the adjoint counter of $\overrightarrow{A_{R}}$ with metric cofficient operator $\Omega$, then $\vec{\pi}_{0,+1}^{\circ}(1)$ and $\vec{\pi}_{-2,-1}^{\circ}(1)$ are left-hand zero spin particles accordingly. we have

$$
{\overrightarrow{A_{L}}}^{\odot}=\overrightarrow{\mathbb{S}}_{0,+1 ;-2,-1}^{\odot}(1)=\vec{\pi}_{0,+1}^{\odot}(1)+\vec{\pi}_{-2,-1}^{\odot}(1)
$$

We are now ready to take further our discussion of scalar product of right-hand-to-right-hand, $\overrightarrow{A_{R}} I_{0} \overrightarrow{A_{R}}$ (11), to psecudo-scalar product of left-hand-to-right-hand, ${\overrightarrow{A_{L}}}^{\ominus} \Omega \overrightarrow{A_{R}}$ (13), as follows

$$
\overrightarrow{A_{R}} I_{0} \overrightarrow{A_{R}} \Rightarrow{\overrightarrow{A_{L}}}^{\ominus} \Omega \overrightarrow{A_{R}}
$$

Here metric cofficient operator $\Omega$ is selected as

$$
\Omega=\left[\begin{array}{lll}
0 & 1 & 0 \\
1 & 0 & 0 \\
0 & 0 & 1
\end{array}\right]
$$

\section{Attention:}

right-hand $0 h$ zero spin particles $\vec{\pi}_{0,+1}(1)$ and $\vec{\pi}_{-2,-1}(1)$ obey angular momentum commutation rules of right-handed coordinate system;

left-hand $0 \hbar$ zero spin particles $\vec{\pi}_{0,+1}^{\odot}(1)$ and $\vec{\pi}_{-2,-1}^{\odot}(1)$ obey angular momentum commutation rules of left-handed coordinate system.

For more concise, the symbols (15) are given in the future discussions $\overrightarrow{A_{R}}(j, k ; r, s) \equiv \vec{\pi}_{j, k}(1)+\vec{\pi}_{r, s}(1), \quad \pi_{3}(i, j) \equiv \pi_{3 ; i, j}(1)$ 
c) Let us have a look at an example of psecudo-scalar product of left-hand-to-right-hand of spin zero particles, ${\overrightarrow{A_{L}}}^{\circ} \Omega \overrightarrow{A_{R}}$. After careful calculation, we get two groups of $0 \hbar$ spin interactions, Group- $\mathrm{A}$ and Group-B.

By way of illustration, we shall refer to the feature $\mathrm{B}(1)$ of Group-B:

Formulas (16.1) (16.2) and formulas (17.1), (17.2) are the third compoments of initial state $i$ and final state $f$ of psecudo-scalar spin interaction of the first generation spin particles $(l=1)$.

Initial state

$$
\begin{array}{ll}
0 \hbar & \pi_{3}(0,+1)=\operatorname{diag}\{\ldots 6,5,4,3,2, \underline{1}, 0,-1,-2,-3,-4, \ldots\} \\
0 \hbar & \pi_{3}(-2,-1)=\operatorname{diag}\{\ldots 4,3,2,1, \underline{0}, \underline{-1},-2,-3,-4,-5,-6 \ldots\}
\end{array}
$$

Final state

$$
\begin{array}{ll}
0 \hbar & \pi_{3}(0,-1)=\operatorname{diag}\{\ldots 5,4,3,2,1, \underline{0},-1,-2,-3,-4,-5, \ldots\} \\
1 \hbar & \pi_{3}(-2,+1)=\operatorname{diag}\{\ldots 5,4,3,2,1, \underline{0},-1,-2,-3,-4,-5, \ldots\}
\end{array}
$$

And the conservation of the third compoment of spin angular momentums between initial state $i$ and final state $f$ is obtained as (18.0)

$$
\begin{aligned}
& \pi_{3}(0,+1 ;-2,-1)=\pi_{3}(0,-1 ;-2,+1) \\
& \text { ininal sum of s.a.m } \quad \text { final sum of s.a.m }
\end{aligned}
$$

Where

$$
\begin{aligned}
& \pi_{3}(0,+1 ;-2,-1)=\pi_{3}(0,+1)+\pi_{3}(-2,-1) \\
& \pi_{3}(0,-1 ;-2,+1)=\pi_{3}(0,-1)+\pi_{3}(-2,+1)
\end{aligned}
$$

And the conservation of psecudo-scalar spin interaction about ininl state $i$ and final state $f$ is obtained as (19.0)

$$
\mathcal{L}_{B, i}(1)=\mathcal{L}_{B, f}(1)=\mathcal{L}_{B}
$$

Where

$$
\begin{aligned}
& \mathcal{L}_{B, i}(1)=\overrightarrow{B_{L, 1}} \oplus(0,+1 ;-2,-1) \Omega \overrightarrow{B_{R, 1}}(0,+1 ;-2,-1) \\
& \mathcal{L}_{B, f}(1)=\overrightarrow{B_{L, 1}}(0,-1 ;-2,+1) \Omega \overrightarrow{B_{R, 1}}(0,-1 ;-2,+1) \\
& \mathcal{L}_{B}=-2 \operatorname{diag}\left\{\ldots 10^{2}, 8^{2}, 6^{2}, 4^{2}, 2^{2}, \underline{0^{2}}, 2^{2}, 4^{2}, 6^{2}, 8^{2}, 10^{2}, \ldots\right\}
\end{aligned}
$$

What mentioned above is so-called fission of $0 \hbar$ zero spin particles, refer to Fig4.

Conservation (18.0) and conservation (19.0) imply that if initial state $i$ and final state $f$ exchange places, so-called fusion of other spin particles is given, refer to Fig3.

Fig1, Fig2 of Group-A are obtained by the analogy to those of Fig3, Fig4 of Group-B above. 


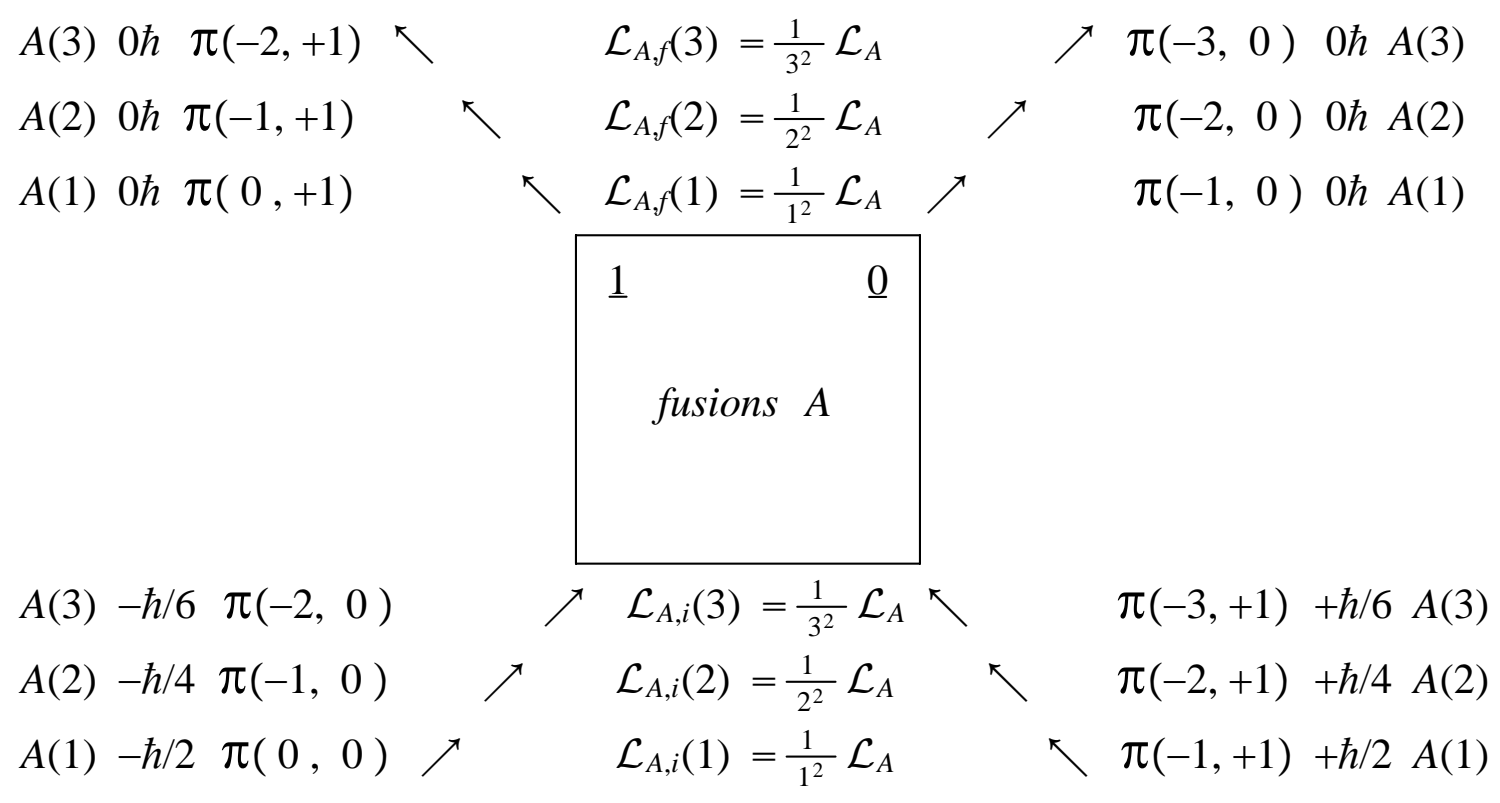

Fig1 zero spin particles are formed by the fusions of other spin particles

$$
\begin{aligned}
& A(3)-\hbar / 6 \pi(-2,0) \nwarrow \quad \mathcal{L}_{A, f}(3)=\frac{1}{3^{2}} \mathcal{L}_{A} \quad \nearrow \pi(-3,+1)+\hbar / 6 A(3) \\
& A(2)-\hbar / 4 \pi(-1,0) \quad \nwarrow \quad \mathcal{L}_{A, f}(2)=\frac{1}{2^{2}} \mathcal{L}_{A} \quad \nearrow \quad \pi(-2,+1)+\hbar / 4 A(2) \\
& A(1)-\hbar / 2 \pi(0,0) \quad \backslash \quad \mathcal{L}_{A, f}(1)=\frac{1}{1^{2}} \mathcal{L}_{A} \nearrow \quad \pi(-1,+1)+\hbar / 2 A(1) \\
& \text { A(3) } 0 \hbar \pi(-2,+1) \quad \nearrow \quad \mathcal{L}_{A, i}(3)=\frac{1}{3^{2}} \mathcal{L}_{A} \nwarrow \quad \pi(-3,0) 0 \hbar A(3) \\
& A(2) 0 \hbar \pi(-1,+1) \quad \nearrow \quad \mathcal{L}_{A, i}(2)=\frac{1}{2^{2}} \mathcal{L}_{A} \quad \backslash \quad \pi(-2,0) 0 \hbar A(2) \\
& A(1) 0 \hbar \pi(0,+1) \quad \nearrow \quad \mathcal{L}_{A, i}(1)=\frac{1}{1^{2}} \mathcal{L}_{A} \quad \backslash \pi(-1,0) 0 \hbar A(1)
\end{aligned}
$$

Fig2 other spin particles are released by the fissions of zero spin particles

$$
\begin{aligned}
& \mathcal{L}_{A, f}(3) \equiv \overrightarrow{A_{L, 3}}(-2,0 ;-3,+1) \Omega \overrightarrow{A_{R, 3}}(-2,-1 ;-3,+1) \\
& \mathcal{L}_{A, i}(3) \equiv{\overrightarrow{A_{L, 3}}}^{\odot}(-2,+1 ;-2,+1) \Omega \overrightarrow{A_{R, 3}}(0,+1 ;-3,0) \\
& \mathcal{L}_{A, f}(2) \equiv \overrightarrow{A_{L, 2}} \stackrel{\odot}{(}(-1,0 ;-2,+1) \Omega \overrightarrow{A_{R, 2}}(-1,-1 ;-2,+1) \\
& \mathcal{L}_{A, i}(2) \equiv \overrightarrow{A_{L, 2}}(-1,+1 ;-1,+1) \Omega \overrightarrow{A_{R, 2}}(-1,+1 ;-2,0) \\
& \mathcal{L}_{A, f}(1) \equiv \overrightarrow{A_{L, 1}}(0,0 ;-1,+1) \Omega \overrightarrow{A_{R, 1}}(0,0 ;-1,+1) \\
& \mathcal{L}_{A, i}(1) \equiv \overrightarrow{A_{L, 1}}(0,+1 ;-1,0) \Omega \overrightarrow{A_{R, 1}}(0,+1 ;-1,0)
\end{aligned}
$$




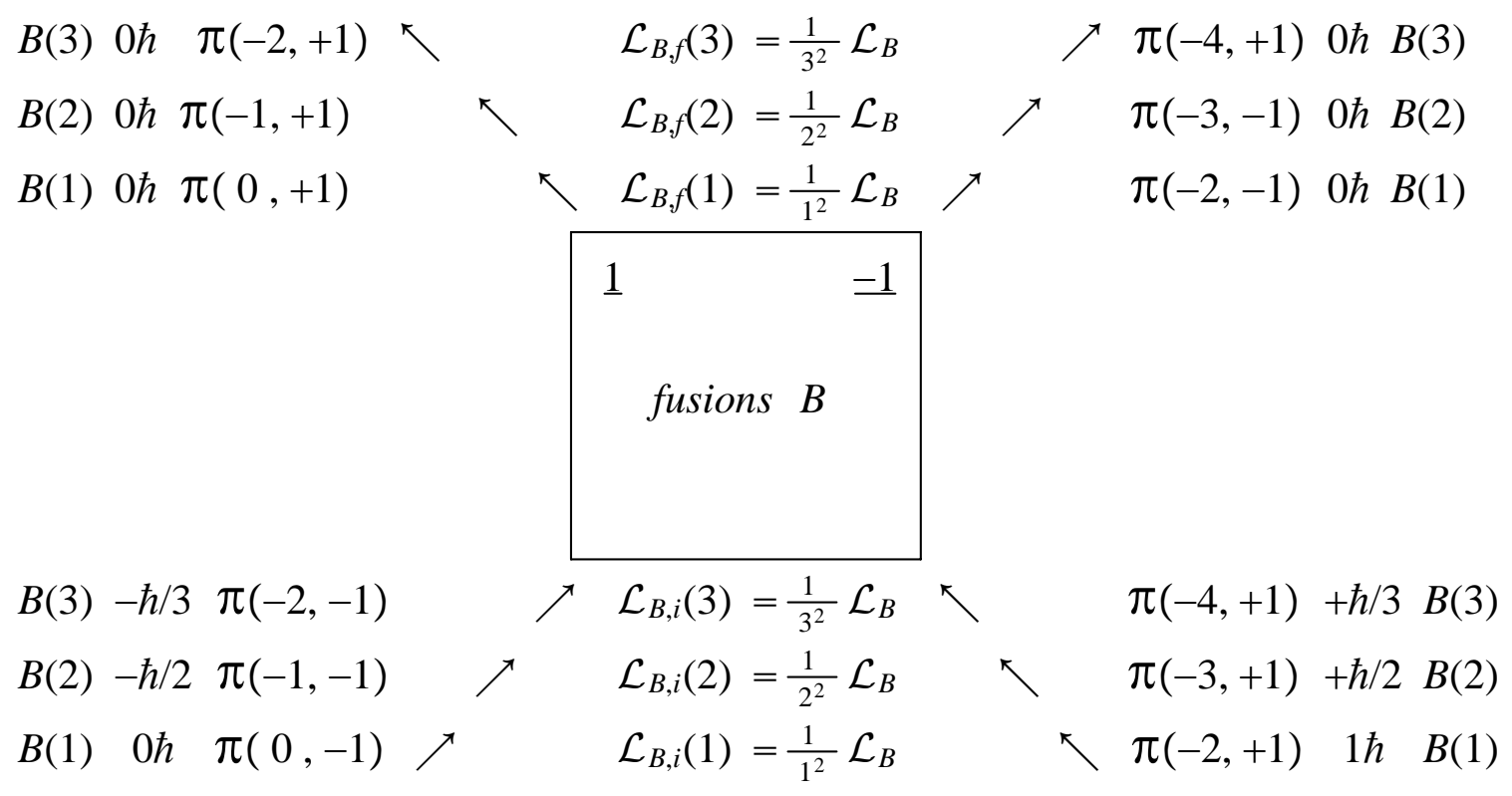

Fig3 zero spin particles are formed by the fusions of other spin particles

$$
\begin{aligned}
& B(3)-\hbar / 3 \pi(-2,-1) \backslash \quad \mathcal{L}_{B, f}(3)=\frac{1}{3^{2}} \mathcal{L}_{B} \quad \nearrow \pi(-4,+1)+\hbar / 3 \quad B(3) \\
& B(2)-\hbar / 2 \pi(-1,-1) \quad \backslash \quad \mathcal{L}_{B, f}(2)=\frac{1}{2^{2}} \mathcal{L}_{B} \quad \nearrow \quad \pi(-3,+1)+\hbar / 2 B(2) \\
& B(1) \quad 0 \hbar \pi(0,-1) \quad \backslash \quad \mathcal{L}_{B, f}(1)=\frac{1}{1^{2}} \mathcal{L}_{B} \nearrow \quad \pi(-2,+1) \quad 1 \hbar^{*} B(1) \\
& B(3) \quad 0 \hbar \pi(-2,+1)
\end{aligned}
$$

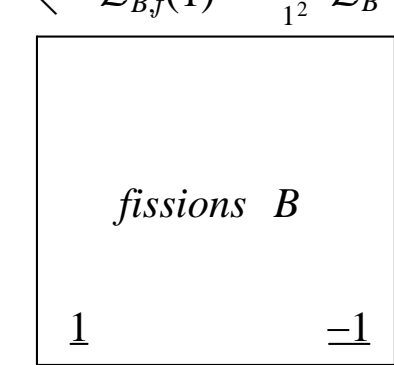

$$
\begin{aligned}
& B(2) \quad 0 \hbar \pi(-1,+1) \\
& \mathcal{L}_{B, i}(3)=\frac{1}{3^{2}} \mathcal{L}_{B} \\
& \pi(-4,-1) \quad 0 \hbar B(3) \\
& B(1) 0 \hbar \pi(0,+1) \\
& \mathcal{L}_{B, i}(2)=\frac{1}{2^{2}} \mathcal{L}_{B} \\
& \pi(-3,-1) \quad 0 \hbar B(2) \\
& \mathcal{L}_{B, i}(1)=\frac{1}{1^{2}} \mathcal{L}_{B} \\
& \nwarrow \pi(-2,-1) \quad 0 \hbar B(1)
\end{aligned}
$$

Fig4 other spin particles are released by the fissions of zero spin particles

$$
\begin{aligned}
& \mathcal{L}_{B, f}(3) \equiv \overrightarrow{B_{L, 3}}(-2,-1 ;-4,+1) \Omega \overrightarrow{B_{R, 3}}(-2,-1 ;-4,+1) \\
& \mathcal{L}_{B, i}(3) \equiv{\overrightarrow{B_{L, 3}}}^{\ominus}(-2,+1 ;-4,-1) \Omega \overrightarrow{B_{R, 3}}(0,+1 ;-4,-1) \\
& \mathcal{L}_{B, f}(2) \equiv \overrightarrow{B_{L, 2}} \stackrel{\odot}{\longrightarrow}(-1,-1 ;-3,+1) \Omega \overrightarrow{B_{R, 2}}(-1,-1 ;-3,+1) \\
& \mathcal{L}_{B, i}(2) \equiv \overrightarrow{B_{L, 2}} \stackrel{\odot}{\longrightarrow}(-1,+1 ;-3,-1) \Omega \overrightarrow{B_{R, 2}}(-1,+1 ;-3,-1) \\
& \mathcal{L}_{B, f}(1) \equiv \overrightarrow{B_{L, 1}}(0,-1 ;-2,+1) \Omega \overrightarrow{B_{R, 1}}(0,-1 ;-2,+1) \\
& \mathcal{L}_{B, i}(1) \equiv \overrightarrow{B_{L, 1}}(0,+1 ;-2,-1) \Omega \overrightarrow{B_{R, 1}}(0,+1 ;-2,-1)
\end{aligned}
$$




\section{4) Spin Interactions between Higgs Bosons and Gravitons}

d) To return to the case of scalar product of right-hand-to-right-hand particles, $\overrightarrow{A_{R}} I \overrightarrow{A_{R}}$, because we could get no-trivial spin-coupling interactions between $0 \hbar$ zero spin particle and $2 \hbar$ spin particle. Here two groups of , Group-C and Group-D. are given. Analogously, illustration by example of the feature $\mathrm{D}(1)$ of Group-D as follows:

Formulas (29.1) (29.2) and formulas (30.1), (30.2) are the third compoments of initial state $i$ and final state $f$ of scalar spin interaction of the first generation spin particles $(l=1)$

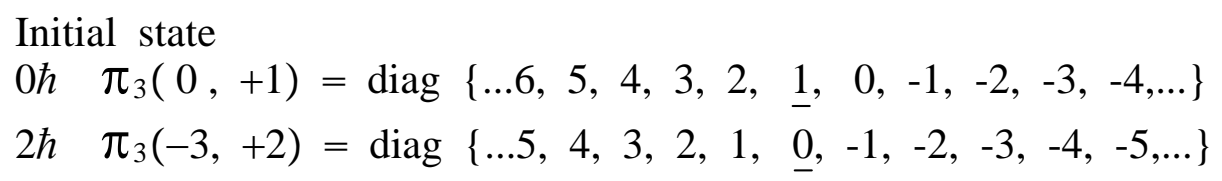

Final state

$1 \hbar / 2 \quad \pi_{3}(0,+2)=\frac{1}{2} \operatorname{diag}\{\ldots 13,11,9,7,5, \quad 3,1,-1,-3,-5,-7, \ldots\}$

$3 \hbar / 2 \quad \pi_{3}(-3,+1)=\frac{1}{2} \operatorname{diag}\{\ldots 9, \quad 7,5,3,1,-1,-3,-5,-7,-9,-11 \ldots\}$

And the conservation of the third compoment of spin angular momentums between initial state $i$ and final state $f$ is obtained as (31.0)

$$
\begin{aligned}
& \pi_{3}(0,+1 ;-3,+2)=\pi_{3}(0,+2 ;-3,+1) \\
& \text { ininal sum of s.a.m } \quad \text { final sum of s.a.m }
\end{aligned}
$$

Where

$$
\begin{aligned}
& \pi_{3}(0,+1 ;-3,+2)=\pi_{3}(0,+1)+\pi_{3}(-3,+2) \\
& \pi_{3}(0,+2 ;-3,+1)=\pi_{3}(0,+2)+\pi_{3}(-3,+1)
\end{aligned}
$$

And the conservation of scalar spin interaction about ininl state $i$ and final state $f$ is obtained as (32.0)

$$
\mathcal{L}_{D, i}(1)=\mathcal{L}_{D, f}(1)=\mathcal{L}_{D}
$$

Where

$$
\begin{aligned}
& \mathcal{L}_{D, i}(1)=\overrightarrow{D_{R, 1}}(0,+1 ;-3,+2) I \overrightarrow{D_{R, 1}}(0,+1 ;-3,+2) \\
& \mathcal{L}_{D, f}(1)=\overrightarrow{D_{R, 1}}(0,+2 ;-3,+1) I \overrightarrow{D_{R, 1}}(0,+2 ;-3,+1) \\
& \mathcal{L}_{D}=8 I_{0}
\end{aligned}
$$

What mentioned above is so-called fission of Higgs Boson and Graviton, refer to Fig8.

Conservation (31.0) and conservation (32.0) imply that if initial state $i$ and final state $f$ exchange places, so-called fusion of other spin particles is given, refer to Fig7.

Fig5, Fig6 of Group-C are obtained by the analogy to those of Fig7, Fig8 of Group-D above. 


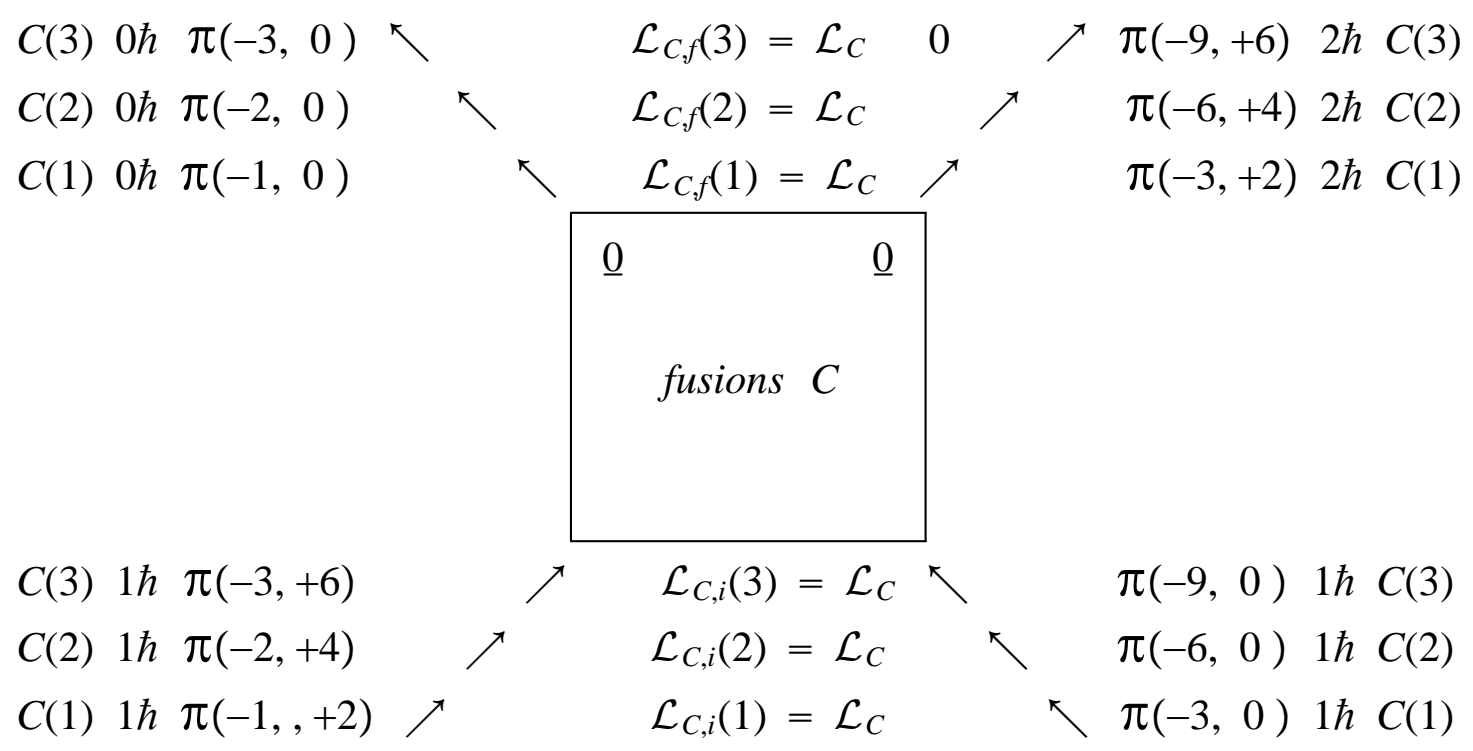

Fig5 Higgs Boson and Graviton are formed by fusions of other spin particles

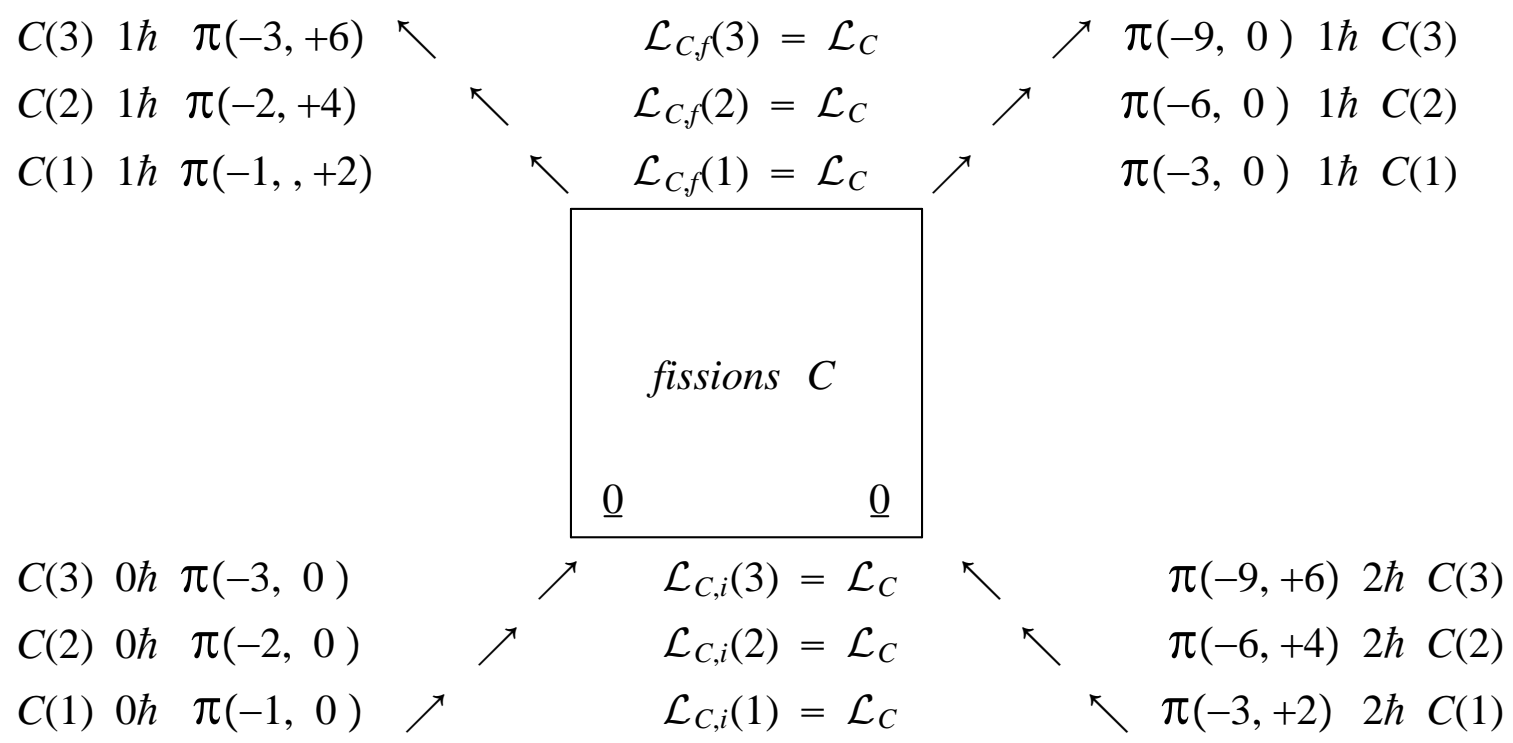

Fig6 other spin particles are released by fissions of Higgs Boson and Graviton

$$
\begin{aligned}
& \mathcal{L}_{C, f}(3) \equiv \overrightarrow{C_{R, 3}}(-3,+6 ;-9,0) I_{0} \overrightarrow{C_{R, 3}}(-3,+6 ;-9,0) \\
& \mathcal{L}_{C, i}(3) \equiv \overrightarrow{C_{R, 3}}(-3,0 ;-9,+6) I_{0} \overrightarrow{C_{R, 3}}(-3,0 ;-9,+6) \\
& \mathcal{L}_{C, f}(2) \equiv \overrightarrow{C_{R, 2}}(-2,+4 ;-6,0) I_{0} \overrightarrow{C_{R, 2}}(-2,+4 ;-6,0) \\
& \mathcal{L}_{C, i}(2) \equiv \overrightarrow{C_{R, 2}}(-2,0 ;-6,+4) I_{0} \overrightarrow{C_{R, 2}}(-2,0 ;-6,+4) \\
& \mathcal{L}_{C, f}(1) \equiv \overrightarrow{C_{R, 1}}(-1,+2 ;-3,0) I_{0} \overrightarrow{C_{R, 1}}(-1,+2 ;-3,0) \\
& \mathcal{L}_{C, i}(1) \equiv{\overrightarrow{C_{R, 1}}}^{\odot}(-1,0 ;-3,+2) I_{0} \overrightarrow{C_{R, 1}}(-1,0 ;-3,+2) \\
& \mathcal{L}_{C}=8 I_{0}
\end{aligned}
$$




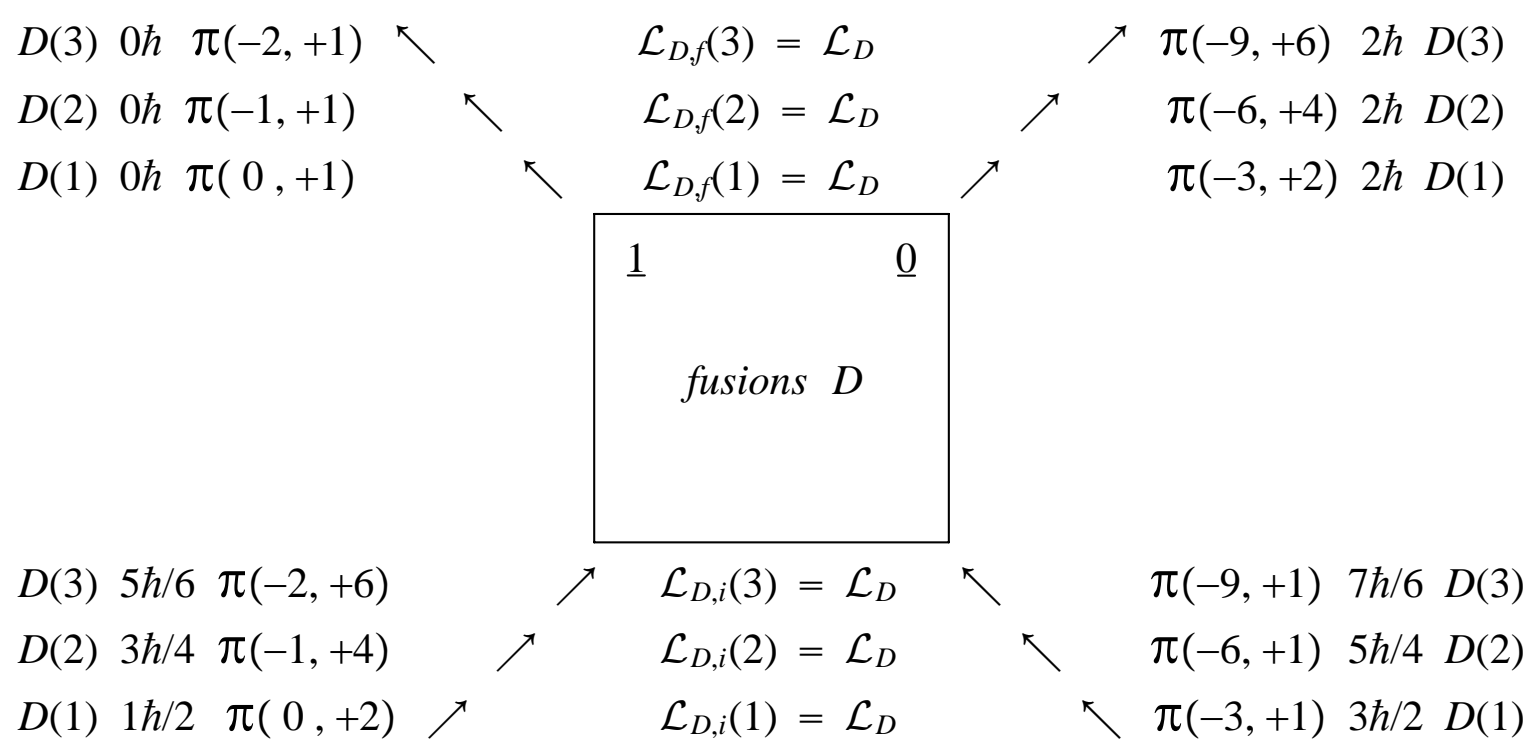

Fig7 Higgs Boson and Graviton are formed by fusions of other spin particles

$$
\begin{aligned}
& D(3) 5 \hbar / 6 \pi(-2,+6) \quad \backslash \quad \mathcal{L}_{D, f}(3)=\mathcal{L}_{D} \quad \nearrow \pi(-9,+1) 7 \hbar / 6 D(3) \\
& D(2) 3 \hbar / 4 \pi(-1,+4) \quad \backslash \quad \mathcal{L}_{D, f}(2)=\mathcal{L}_{D} \quad \nearrow \quad \pi(-6,+1) \quad 5 \hbar / 4 D(2) \\
& D(1) 1 \hbar / 2 \pi(0,+2) \quad \backslash \quad \mathcal{L}_{D, f}(1)=\mathcal{L}_{D} \quad \nearrow \quad \pi(-3,+1) 3 \hbar / 2 D(1)
\end{aligned}
$$

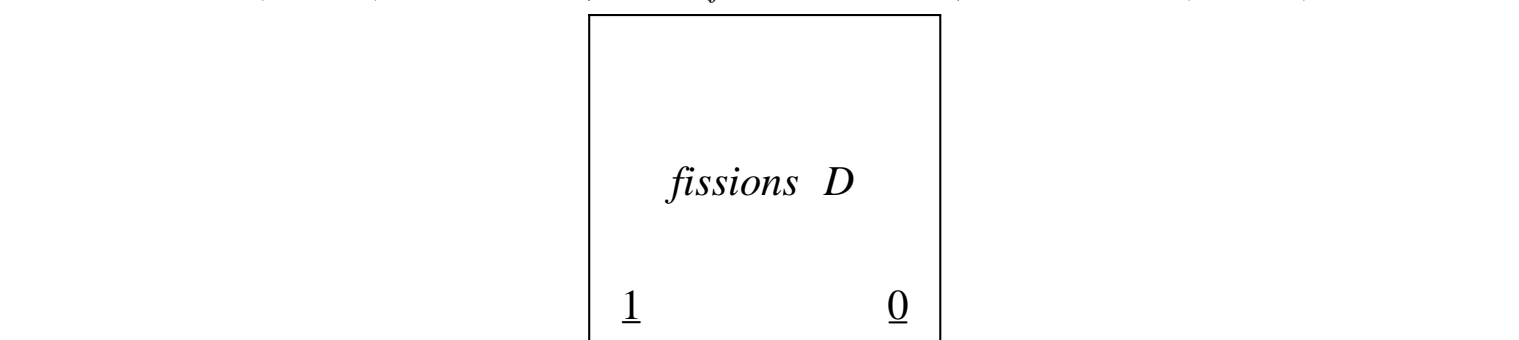

$$
\begin{aligned}
& D(3) 0 \hbar \pi(-2,+1) \quad \nearrow \quad \mathcal{L}_{D, i}(3)=\mathcal{L}_{D} \quad \nwarrow \quad \pi(-9,+6) 2 \hbar \quad D(3) \\
& D(2) 0 \hbar \pi(-1,+1) \quad \nearrow \quad \mathcal{L}_{D, i}(2)=\mathcal{L}_{D} \quad \backslash \quad \pi(-6,+4) 2 \hbar D(2) \\
& D(1) 0 \hbar \pi(0,+1) \quad \nearrow \quad \mathcal{L}_{D, i}(1)=\mathcal{L}_{D} \quad \backslash \pi(-3,+2) 2 \hbar D(1)
\end{aligned}
$$

Fig8 other spin particles are released by fissions of Higgs Boson and Graviton

$$
\begin{aligned}
& \mathcal{L}_{D, f}(3) \equiv{\overrightarrow{D_{R, 3}}}^{\odot}(-2,+6 ;-9,+1) I_{0} \overrightarrow{D_{R, 3}}(-2,+6 ;-9,+1) \\
& \mathcal{L}_{D, i}(3) \equiv \overrightarrow{D_{R, 3}}(-2,+1 ;-9,+6) I_{0} \overrightarrow{D_{R, 3}}(-2,+1 ;-9,+6) \\
& \mathcal{L}_{D, f}(2) \equiv \overrightarrow{D_{R, 2}}(-1,+4 ;-6,+1) I_{0} \quad \overrightarrow{D_{R, 2}}(-1,+4 ;-6,+1) \\
& \mathcal{L}_{D, i}(2) \equiv \overrightarrow{D_{R, 2}}(-1,+1 ;-6,+4) I_{0} \quad \overrightarrow{D_{R, 2}}(-1,+1 ;-6,+4)
\end{aligned}
$$

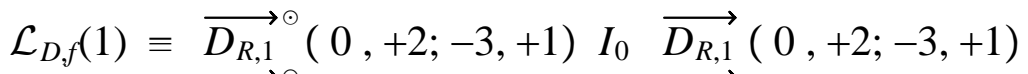

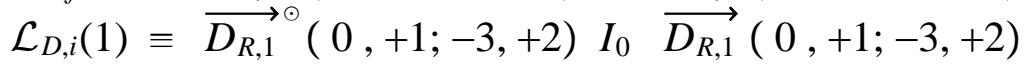

$$
\begin{aligned}
& \mathcal{L}_{D}=8 I_{0}
\end{aligned}
$$




\section{5) Conclusions}

$\mathcal{L}_{A}(l)$ and $\mathcal{L}_{B}(l)$ in paragraph 3 ), which construct self-actions of zero spin particles, could be thought of as the Lagrangian function of Higgs Boson in quantum quantum field. Further research could show that such kind of mechanism may lead to the change of symmetry breaking in the Standard Model.

$\mathcal{L}_{C}(l) \mathcal{L}_{D}(l)$ in paragraph 4), which construct creation and annihilation between $0 h$ zero spin particles and $2 \hbar$ spin particle, may be able to dectect the existent of graviton from the 'particulate' nature of gravitation experimentally, comparative study, wavelike properties of gravitation have been exhibited [5]

\section{References}

[1] Higgs, Peter

Broken Symmetries and the Masses of Gauge Bosons Physical

Review Letters 13 (16) 508-509

Bibcode:1964 PhRvL..13..508H. doi:10.1103/PhysRevLett. 13.508 (1964)

European particle physics laboratory, CERN, (2012)

[2] ShaoXu Ren

Advanced Non-Euclidean Quantum Mechanics

ISBN 978-7-80703-585-4 (2006)

The Third Kind of Particles

ISBN 978-7-900500-91-5 (2011)

ISBN 978-988-15598-9-0 (2012)

ISBN 988-3-659-17892-4 (2012)

The Third Kind of Particles

Journal of Modern Physics, 5, 800-869

http:/dx.doi.org/10.4236/jmp.2014.59090

[3] ShaoXu Ren

The Origins Of Spins Of Elementary Particles

ISBN 978-988-13649-7-5 (2014)

The Origins of Bosons and Fermions

Journal of Modern Physics, 5, 1848-1879

http:/dx.doi.org/10.4236/jmp.2014.517181

[4] ShaoXu Ren

Interaction of the Origins of Spin Angular Momentum

ISBN 978-988-14902-0-9 (2016 2nd edition)

[5] LIGO, LSC (2016)

[6] ShaoXu Ren

Faster Than Velocity Of Light ( Infinite Dimensional Lorentz Group Of TKP )

ISBN 978-988-12266-2-4 (2013) 


\section{6) Appendix: Higgs Boson Wave Differential Equation of First Order} and Klein-Gordon Wave Differential Equation

e) Using math elements $\vec{\pi}_{j, k}$ in STS [4], the Hamiltonians of the first order and the second order linear wave differential equantions of $0 h$ spin zero particles, (Higgs Boson) are written as the following:

$$
\begin{aligned}
& \mathbb{H}_{j, j+l}(l)=\left\{H_{j, j+l}(l)+\rho_{3} m\right\} \\
& \mathbb{H}_{j, j+l ; k, k+l}(l)=\mathbb{H}_{j, j+l}(l) \mathbb{H}_{k, k+l}(l)
\end{aligned}
$$

$H_{j, j+l}(l)$ in (A-1) is kinectic energy. There are many combinations in (A-2), which made by various choise of $j$ and $k$.

For clarity, here $l=1$ and omitting the mark "(1) " in above expressions. Then, taking $j=k=-1$ in cases of (A-1) and (A-2). getting below:

For $(\mathrm{A}-1)$

$$
H_{-1,0}=\rho_{1} \vec{\pi}_{-1,0} \cdot \vec{P}
$$

and first order wave differential equantions of $0 \hbar$ spin zero particle

$0 \hbar$

$$
\left\{i \partial_{t}-\rho_{1} \vec{\pi}_{-1,0} \cdot \vec{P}-\rho_{3} m\right\} \Psi_{-1,0}=0
$$

For $(\mathrm{A}-2)$

$$
\mathbb{H}_{-1,0 ;-1,0}=H_{-1,0}^{2}+m^{2}
$$

and second order wave differential equantions of $0 \hbar$ spin zero particle

$0 \hbar$

$$
\left\{\partial_{t t}^{2}+H_{-1,0}^{2}+m^{2}\right\} \Phi_{-1,0}=0
$$

To make it clearer, we consinder the diagonal terms of (A-5) and have:

$$
\text { diagonal }\left\{H_{-1,0}^{2}\right\}
$$

$=\operatorname{diag}\{,-25 / 2,-16 / 2,-9 / 2,-4 / 2,-1 / 2,0,-1 / 2,-4 / 2,-9 / 2,-16 / 2,-25 / 2,\} P_{1}^{2}$

$+\operatorname{diag}\{,-25 / 2,-16 / 2,-9 / 2,-4 / 2,-1 / 2, \quad 0,-1 / 2,-4 / 2,-9 / 2,-16 / 2,-25 / 2,\} P_{2}^{2}$

$+\operatorname{diag}\{, \quad 25, \quad 16, \quad 9, \quad 4, \quad 1, \quad \underline{0}, \quad 1, \quad 4, \quad 9, \quad 16, \quad 25, \quad\} P_{3}^{2}$

$=\left\{-\frac{1}{2}\left\{P_{1}^{2}+P_{2}^{2}\right\}+P_{3}^{2}\right\} \pi_{0}^{2}(0)$

$\pi_{0}^{2}(0)=\operatorname{diag}\left\{, 5^{2}, 4^{2}, 3^{2}, 2^{2}, 1^{2}, \underline{0^{2}}, 1^{2}, 2^{2}, 3^{2}, 4^{2}, 5^{2},\right\}(\mathrm{A}-10)$

(A-9) indicates

$$
\left\{-\frac{1}{2}\left\{P_{1}^{2}+P_{2}^{2}\right\}+P_{3}^{2}\right\} \subset \text { diagonal }\left\{H_{-1,0}^{2} ; 0 \hbar \text { spin }\right\}(\mathrm{A}-11)
$$
and

$$
\left\{\partial_{t t}^{2}+\frac{1}{2}\left\{\partial_{x x}^{2}+\partial_{y y}^{2}\right\}-\partial_{z z}^{2}+m^{2}\right\} \Phi_{\text {diagonal; }-1,0}=0
$$


f) The Hamiltonians of the first order and the second order linear differential equantions of $\hbar / 2$ spin Fermion particles are written as following:

$$
\begin{aligned}
& \mathbb{H}_{j, j-2 l}(l)=\left\{H_{j, j-2 l}(l)+\rho_{3} m\right\} \\
& \mathbb{H}_{j, j-2 l ; k, k-2 l}(l)=\mathbb{H}_{j, j-2 l}(l) \mathbb{H}_{k, k-2 l}(l)
\end{aligned}
$$

Accordingly, taking $j=k=0$ in case of (A-13) and (A-14), we get:

For (A-13)

$$
H_{0,-2}=2 \rho_{1} \vec{\pi}_{0,-2} \cdot \vec{P}
$$

and first order wave differential equantions of $\hbar / 2$ spin particle

$$
\hbar / 2 \quad\left\{i \partial_{t}-2 \rho_{1} \vec{\pi}_{0,-2} \cdot \vec{P}-\rho_{3} m\right\} \Psi_{0,-2}=0
$$

For (A-14) $\quad \mathbb{H}_{0,-2 ; 0,-2}=H_{0,-2}^{2}+m^{2}$

and second order wave differential equantions of $\hbar / 2$ spin particle

$\hbar / 2$

$$
\left\{\partial_{t t}^{2}+H_{0,-2}^{2}+m^{2}\right\} \Phi_{0,-2}=0
$$

Taking out the diagonal terms from (A-17) and have:

diagonal $\left\{H_{0,-2}^{2}\right\}$

$$
\begin{aligned}
& =\operatorname{diag}\{,-39,-23,-11,-3, \quad \mathbf{1}, \mathbf{1},-3,-11,-23,-39,-59,\} P_{1}^{2} \\
& +\operatorname{diag}\{,-39,-23,-11,-3, \mathbf{1}, \mathbf{1},-3,-11,-23,-39,-59,\} P_{2}^{2} \\
& +\operatorname{diag}\{, \quad 81,49, \quad 25,9, \quad \mathbf{1}, \mathbf{1}, 9, \quad 25, \quad 49,81,121,\} P_{3}^{2}
\end{aligned}
$$

Now, we see the two terms (A-21) in the center part of diagonal $\left\{H_{0,-2}^{2}\right\}$ (A-20), is just the square sum $H_{\text {Dirac }}^{2}$ of kinectic energy of well-known Dirac equation of second order.

$$
\left\{H_{\text {Dirac }}^{2}\right\}=\operatorname{diag}\{\ldots, \mathbf{1}, \quad \underline{\mathbf{1}}, \ldots\}\left\{P_{1}^{2}+P_{2}^{2}+P_{3}^{2}\right\} \subset \text { diagonal }\left\{H_{0,-2}^{2}\right\}
$$

Or

$$
\left\{H_{\text {Dirac }}^{2}\right\}=\left\{H_{K G}\right\}=-\nabla^{2} \subset \text { diagonal }\left\{H_{0,-2}^{2} ; \hbar / 2 \text { spin }\right\}
$$

And contrast with (A-11), we get

$$
\left\{H_{\text {Dirac }}^{2}\right\}=\left\{H_{K G}\right\}=-\nabla^{2} \subsetneq \text { diagonal }\left\{H_{-1,0}^{2} ; 0 \hbar \text { spin }\right\}
$$

Formula (A-22) and (A-23) mean: $-\nabla^{2}$ is a subset of $H_{0,-2}^{2}$, not a set of $H_{-1,0}^{2}$. So Klein-Gordon Equation

$$
\begin{gathered}
\left\{\hbar^{2} \partial_{t t}^{2}-c^{2} \hbar^{2} \nabla^{2}+m^{2} c^{4}\right\} \Phi_{\mathrm{KG}}=0 \\
\left\{\quad \square-x^{2}\right\} \Phi_{\mathrm{KG}}=0 \\
\square=\nabla^{2}-\partial_{t t}^{2} / c^{2}, \quad \chi=m c / \hbar
\end{gathered}
$$

is closer to $\hbar / 2$ spin Fermion particle, rather than $0 \hbar$ spin Boson particle.

It is more reasonable to use equation (A-4), equation (A-6) to describe zero spin Boson particle (Higgs Boson) than to use Klein Gordon equation. 
g) For Vacuum Spin particle, VSP, $-\hbar / 2$ negative one-second fermion particle, its Hamiltonians of the first order and the second order linear wave differential equantions are written as the following:

$$
\begin{aligned}
& \mathbb{H}_{j, j}(l)=\left\{H_{j, j}(l)+\rho_{3} m\right\} \\
& \mathbb{H}_{j, j ; k, k}(l)=\mathbb{H}_{j, j}(l) \mathbb{H}_{k, k}(l)
\end{aligned}
$$

Taking $j=k=0$ in case of (A-27) and (A-28), we get:

For (A-27)

$$
H_{0,0}=2 \rho_{1} \vec{\pi}_{0,0} \cdot \vec{P}
$$

and first order wave differential equantions of $-\hbar / 2$ spin particle

$$
-\hbar / 2 \quad\left\{i \partial_{t}-2 \rho_{1} \vec{\pi}_{0,0} \cdot \vec{P}-\rho_{3} m\right\} \Psi_{0,0 ; \mathrm{VSP}}=0
$$

For (A-28) $\quad \mathbb{H}_{0,0 ; 0,0}=H_{0,0}^{2}+m^{2}$

and second order wave differential equantions of $-\hbar / 2$ spin particle

$-\hbar / 2$

$$
\left\{\partial_{t t}^{2}+H_{0,0}^{2}+m^{2}\right\} \Phi_{0,0 ; \mathrm{VSP}}=0
$$

Taking out the diagonal terms from (A-31) and have: diagonal $\left\{H_{0,0}^{2}\right\}$

$=\operatorname{diag}\{,-61,-41,-25,-13,-5, \underline{-\mathbf{1}}, \underline{-\mathbf{1}},-5,-13,-25,-41,\} P_{1}^{2}$

$+\operatorname{diag}\{,-61,-41,-25,-13,-5,-\mathbf{- 1},-\mathbf{1},-5,-13,-25,-41,\} P_{2}^{2}$

$+\operatorname{diag}\{, 121,81,49,25,9, \mathbf{1}, \mathbf{1}, 9,25,49,81,\} P_{3}^{2}$

Now turn to the two terms (A-35) in the center part of diagonal $\left\{H_{0,0}^{2}\right\}$

$$
\left\{H_{\mathrm{VSP}}\right\}=\operatorname{diag}\{\ldots, \quad \underline{\mathbf{1}}, \mathbf{1}, \ldots\}\left\{-P_{1}^{2}-P_{2}^{2}+P_{3}^{2}\right\} \subset \text { diagonal }\left\{H_{0,0}^{2}\right\}
$$

and have wave equation of VSP, (- $\hbar / 2$ spin fermion particle)

$$
\left\{\partial_{t t}^{2}+\partial_{x x}^{2}+\partial_{y y}^{2}-\partial_{z z}^{2}+m^{2}\right\} \Phi_{\mathrm{VSP}}=0
$$

h) Next we shall discuss the solutions $\Psi^{0} \equiv \Psi_{j, 0}^{m=0}$ of zero mass particle differential equantions of First Order, which are based on free $-\hbar / 2$ VSP particle (A-30), free $0 \hbar$ zero spin particle (Higgs Boson) (A-4), free $+\hbar / 2$ Dirac spin particle (A-16). Which are given as below:

$$
\begin{array}{ll}
-\hbar / 2 & \left\{i \partial_{t}-2 \vec{\pi}_{0,0} \cdot \vec{P}\right\} \Psi_{0,0 ; \mathrm{VSP}}^{0}=0 \\
0 \hbar & \left\{i \partial_{t}-\vec{\pi}_{-1,0} \cdot \vec{P}\right\} \Psi_{-1,0 ; \text { Higgs Boson }}^{0}=0 \\
+\hbar / 2 & \left\{i \partial_{t}-2 \vec{\pi}_{0,-2} \cdot \vec{P}\right\} \Psi_{0,-2 ; \text { Dirac }}^{0}=0
\end{array}
$$


Notation:

$$
E=E_{ \pm}= \pm p, p=\sqrt{p_{1}^{2}+p_{2}^{2}+p_{3}^{2}}
$$

h1) For free $-\hbar / 2$ VSP zero mass particle (A-37)

Getting

$$
\Psi_{0,0 ; \mathrm{VSP}}^{0}=F_{0,0} e^{-i E t}
$$

$$
\begin{aligned}
& F_{+p ; 0,0} e^{-i E_{+} t} \\
& =\left[\begin{array}{lll}
-\left(p-p_{3}\right)^{5} & / p_{+}^{5} \\
+\left(p-p_{3}\right)^{4} & / p_{+}^{4} \\
-\left(p-p_{3}\right)^{3} & / p_{+}^{3} \\
+\left(p-p_{3}\right)^{2} & / p_{+}^{2} \\
-\left(p-p_{3}\right)^{1} & / p_{+}^{1} \\
+\left(p-p_{3}\right)^{0} & / p_{+}^{0} \\
+\left(p+p_{3}\right)^{0} & / p_{-}^{0} \\
-\left(p+p_{3}\right)^{1} & / p_{-}^{1} \\
+\left(p+p_{3}\right)^{2} & / p_{-}^{2} \\
-\left(p+p_{3}\right)^{3} & / p_{-}^{3} \\
+\left(p+p_{3}\right)^{4} & / p_{-}^{4} \\
-\left(p+p_{3}\right)^{5} & / p_{-}^{5}
\end{array}\right] e^{-i E_{+} t}
\end{aligned}
$$

(A-41.1)

$$
=\left[\begin{array}{lll} 
& F_{-p ; 0,0} e^{-i E_{-} t} \\
+ & \left(p+p_{3}\right)^{5} & / p_{+}^{5} \\
+ & \left(p+p_{3}\right)^{4} & / p_{+}^{4} \\
+ & \left(p+p_{3}\right)^{3} & / p_{+}^{3} \\
+ & \left(p+p_{3}\right)^{2} & / p_{+}^{2} \\
+ & \left(p+p_{3}\right)^{1} & / p_{+}^{1} \\
+ & \left(p+p_{3}\right)^{0} & / p_{+}^{0} \\
+ & \left(p-p_{3}\right)^{0} & / p_{-}^{0} \\
+ & \left(p-p_{3}\right)^{1} & / p_{-}^{1} \\
+ & \left(p-p_{3}\right)^{2} & / p_{-}^{2} \\
+ & \left(p-p_{3}\right)^{3} & / p_{-}^{3} \\
+ & \left(p-p_{3}\right)^{4} & / p_{-}^{4} \\
+ & \left(p-p_{3}\right)^{5} & / p_{-}^{5}
\end{array}\right] e^{-i E_{-} t}
$$

(A-41.2)

$F_{+p ; \theta>0}=\left[\begin{array}{lll}- & \tan ^{5} \theta / 2 & e^{-i 5 \varphi} \\ + & \tan ^{4} \theta / 2 & e^{-i 4 \varphi} \\ - & \tan ^{3} \theta / 2 & e^{-i 3 \varphi} \\ + & \tan ^{2} \theta / 2 & e^{-i 2 \varphi} \\ - & \tan ^{1} \theta / 2 & e^{-i \varphi} \\ + & & e^{+i 0 \varphi}\end{array}\right]$
$F_{+p ; \theta<0}=\left[\begin{array}{lll}+ & & e^{+i 0 \varphi} \\ - & \cot ^{1} \theta / 2 & e^{+i \varphi} \\ + & \cot ^{2} \theta / 2 & e^{+i 2 \varphi} \\ - & \cot ^{3} \theta / 2 & e^{+i 3 \varphi} \\ + & \cot ^{4} \theta / 2 & e^{+i 4 \varphi} \\ - & \cot ^{5} \theta / 2 & e^{+i 5 \varphi}\end{array}\right]$

(A-41.3)

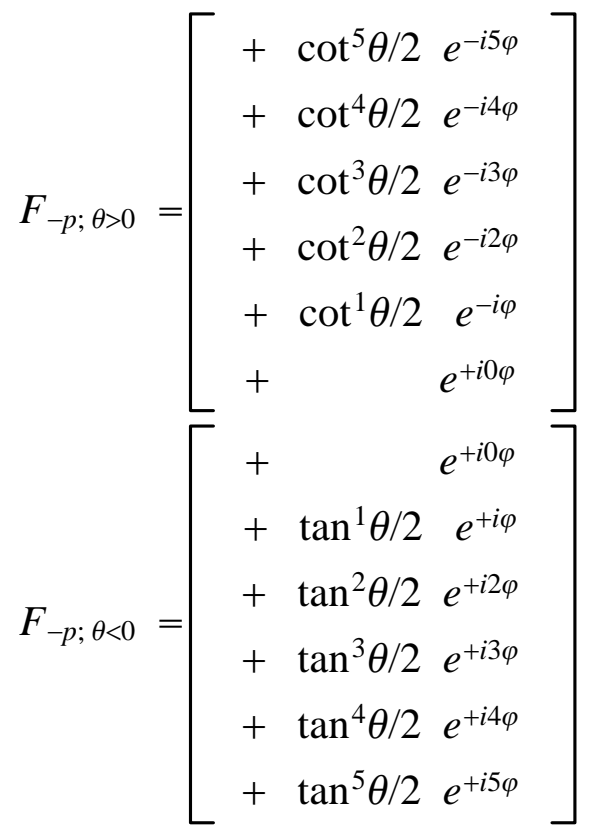

(A-41.4)

VSP particle $F_{+p ; 0,0}=F_{+p ; \theta>0}+F_{+p ; \theta<0}$

$$
F_{-p ; 0,0}=F_{-p ; \theta>0}+F_{-p ; \theta<0}
$$


h2) For free $0 h$ zero spin zero mass particle (Higgs Boson) (A-38)

$$
\Psi_{-1,0 ; \mathrm{HB}}^{0}=F_{-1,0} e^{-i E t}
$$

Getting

$$
=\left[\begin{array}{l}
\Psi_{+p ;-1,0 ; \mathrm{HB}}^{0}-\left(p-p_{3}\right)^{4} p / p_{+}^{5} \\
+\left(p-p_{3}\right)^{3} p / p_{+}^{4} \\
-\left(p-p_{3}\right)^{2} p / p_{+}^{3} \\
+\left(p-p_{3}\right)^{1} p / p_{+}^{2} \\
-\left(p-p_{3}\right)^{0} p / p_{+}^{1} \\
+\left(p \mp p_{3}\right)^{0} p^{0} / p_{ \pm}^{0} \\
-\left(p+p_{3}\right)^{0} p / p_{-}^{1} \\
+\left(p+p_{3}\right)^{1} p / p_{-}^{2} \\
-\left(p+p_{3}\right)^{2} p / p_{-}^{3} \\
+\left(p+p_{3}\right)^{3} p / p_{-}^{4} \\
-\left(p+p_{3}\right)^{4} p / p_{-}^{5}
\end{array}\right] e^{-i E_{+} t}
$$$$
(\mathrm{A}-42.1)
$$$$
=\left[\begin{array}{ccc}
- & \tan ^{4} \theta / 2 & e^{-i 5 \varphi} \\
+ & \tan ^{3} \theta / 2 & e^{-i 4 \varphi} \\
- & \tan ^{2} \theta / 2 & e^{-i 3 \varphi} \\
+ & \tan ^{1} \theta / 2 & e^{-i 2 \varphi} \\
- & & e^{-i \varphi} \\
& \sin \theta & \\
- & & e^{+i \varphi} \\
+ & \cot \theta^{1} / 2 & e^{+i 2 \varphi} \\
- & \cot \theta^{2} / 2 & e^{+i 3 \varphi} \\
+ & \cot \theta^{3} / 2 & e^{+i 4 \varphi} \\
- & \cot \theta^{4} / 2 & e^{+i 5 \varphi}
\end{array}\right] e^{-i E_{+} t}
$$

$(\mathrm{A}-42.3)$

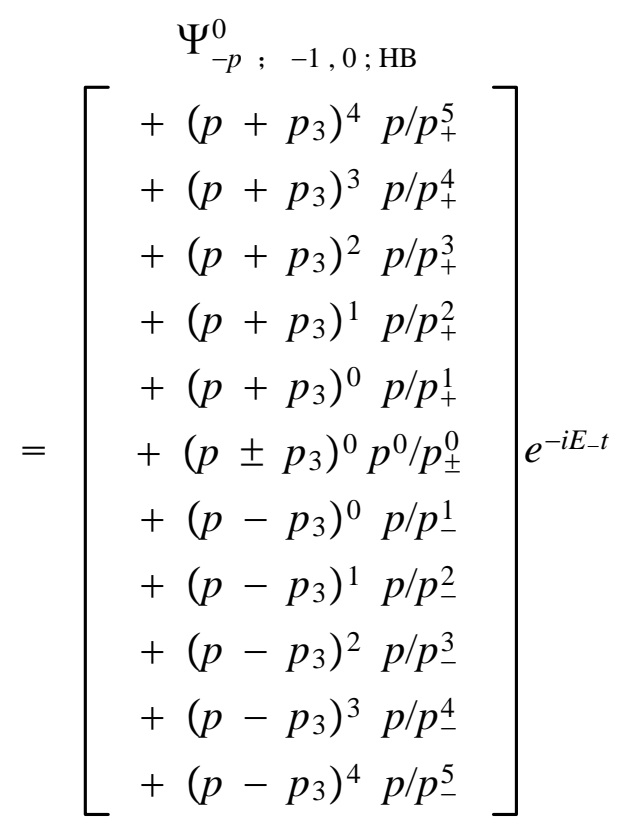

$(\mathrm{A}-42.2)$

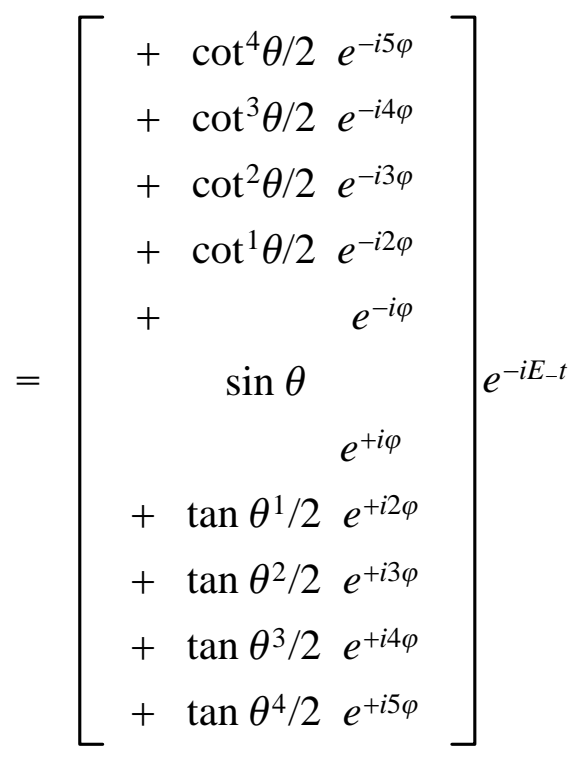

$(\mathrm{A}-42.4)$

There are two singularities at $\theta=0$, and $\pi$ in the above two expressions. Obviously, some uncertainties of choise of free $0 h$ zero spin zero mass wavefunction should be addressed. Here (A-42.3) and (A-42.4) are only an investigation. 
h3) For free $+\hbar / 2$ Dirac spin zero mass particle (A-39)

Getting

$$
\Psi_{0,-2 ; \text { Dirac }}^{0}=F_{0,-2} e^{-i E t}
$$

$$
\begin{aligned}
& \Psi_{+p}^{0} ; 0,-2 \text {; Dirac } \\
& =\left[\begin{array}{c}
\ldots \ldots \\
+\left(p+p_{3}\right)^{4} / p_{+}^{4} \\
+\left(p+p_{3}\right)^{3} / p_{+}^{3} \\
+\left(p+p_{3}\right)^{2} / p_{+}^{2} \\
+\left(p+p_{3}\right)^{1} / p_{+}^{1} \\
+\left(p \pm p_{3}\right)^{0} / p_{ \pm}^{0} \\
+\left(p-p_{3}\right)^{1} / p_{-}^{1} \\
+\left(p-p_{3}\right)^{2} / p_{-}^{2} \\
+\left(p-p_{3}\right)^{3} / p_{-}^{3} \\
+\left(p-p_{3}\right)^{4} / p_{-}^{4} \\
+\left(p-p_{3}\right)^{5} / p_{-}^{5} \\
\ldots . .
\end{array}\right] e^{-i E_{+} t} \\
& \text { (A-43.1) } \\
& =\left[\begin{array}{ll} 
& \ldots . . \\
\cot ^{4} \theta / 2 & \cos \theta / 2 e^{-i 4 \varphi} \\
\cot ^{3} \theta / 2 & \cos \theta / 2 e^{-i 3 \varphi} \\
\cot ^{2} \theta / 2 & \cos \theta / 2 e^{-i 2 \varphi} \\
\cot ^{1} \theta / 2 & \cos \theta / 2 e^{-i \varphi} \\
& \cos \theta / 2 \\
& \sin \theta / 2 e^{+i \varphi} \\
\tan ^{1} \theta / 2 & \sin \theta / 2 e^{+i 2 \varphi} \\
\tan ^{2} \theta / 2 & \sin \theta / 2 e^{+i 3 \varphi} \\
\tan ^{3} \theta / 2 & \sin \theta / 2 e^{+i 4 \varphi} \\
\tan ^{4} \theta / 2 & \sin \theta / 2 e^{+i 5 \varphi} \\
& \cdots . .
\end{array}\right] e^{-i E_{+} t},= \\
& \Psi_{-p}^{0} ; 0,-2 \text {; Dirac } \\
& -\left(p-p_{3}\right)^{5} / p_{+}^{5} \\
& +\left(p-p_{3}\right)^{4} / p_{+}^{4} \\
& -\left(p-p_{3}\right)^{3} / p_{+}^{3} \\
& +\left(p-p_{3}\right)^{2} / p_{+}^{2} \\
& -\left(p-p_{3}\right)^{1} / p_{+}^{1} \\
& +\left(p \mp p_{3}\right)^{0} / p_{ \pm}^{0} \\
& -\left(p+p_{3}\right)^{1} / p_{-}^{1} \\
& +\left(p+p_{3}\right)^{2} / p_{-}^{2} \\
& -\left(p+p_{3}\right)^{3} / p_{-}^{3} \\
& +\left(p+p_{3}\right)^{4} / p_{-}^{4} \\
& \cos \theta / 2
\end{aligned}
$$

\section{$(\mathrm{A}-43.2)$}

(A-43.3)

(A-43.4)

The two elements in the centers of the above expressions are just the spin wavefunction representation of operator $\vec{\sigma} \cdot \vec{n}$ of $\hbar / 2$ Dirac spin in two dimensional spin space in traditional quantum machenics.

$$
=\left[\begin{array}{c}
\Psi_{+p ; \text { Dirac }}^{0} \cos \theta / 2 \\
\sin \theta / 2 e^{+i \varphi}
\end{array}\right], \quad=\left[\begin{array}{c}
\Psi_{-p ; \text { Dirac }}^{0} \\
-\sin \theta / 2 e^{-i \varphi} \\
\cos \theta / 2 \\
(\mathrm{~A}-43.5)
\end{array}\right]
$$


i) Finally we digress slightly, to tackle the situations of the velocity of light, because light speed is related to spin angular momentum in STS.

i1) The special case of $P_{1}=P_{1}=0$ for non-zero mass particle Second Order differential equantions $-\hbar / 2$ (A-32), VSP particle, zero spin particle (Higgs Boson) (A-6), $+\hbar / 2$ Dirac spin particle (A-18) are given below:

$$
\begin{aligned}
& -\hbar / 2 \quad\left\{E^{2}+\alpha_{0,0}^{2} \partial_{z z}^{2}-m^{2}\right\} \Phi_{0,0 ; \mathrm{VSP}}=0 \\
& \alpha_{0,0}^{2}=\operatorname{diag}\{, 121,81,49,25,9, \mathbf{1}, \mathbf{1}, 9,25,49,81,\} \\
& 0 \hbar\left\{E^{2}+\alpha_{-1,0}^{2} \partial_{z z}^{2}-m^{2}\right\} \Phi_{-1,0 ; \text { Higgs Boson }}=0 \\
& \alpha_{-1,0}^{2}=\operatorname{diag}\{, 25, \quad 16, \quad 9, \quad 4, \mathbf{1}, \quad \underline{0}, \mathbf{1}, 4, \quad 9,16,25,\} \\
& +\hbar / 2 \quad\left\{E^{2}+\alpha_{0,-2}^{2} \partial_{z z}^{2}-m^{2}\right\} \Phi_{0,-2 ; \text { Dirac }}=0 \\
& \alpha_{0,-2}^{2}=\operatorname{diag}\{, 81,49,25,9, \mathbf{1}, \mathbf{1}, 9,25,49,81,121,\}
\end{aligned}
$$

getting [2]

$$
E_{S=|j-k|}^{ \pm}= \pm \sqrt{m^{2} c^{4}+\alpha_{i, j}^{2} c^{2} P_{3}^{2}} \geq \sqrt{m^{2} c^{4}+c^{2} P_{3}^{2}}
$$

Photon velocity in multi-level universes world is quantized: the limiting speed of particle with zero mass $m=0$, could be greater than $c$

$$
C_{S=|j-k|}=\alpha_{i, j} c=1 c, 2 c, 3 c, 4 c, \ldots \text { or } 1 c, 3 c, 5 c, 7 c, \ldots \geq c,
$$

i2) Lorentz Group Operators are constructed by six $4 \times 4$ dimensional matrices : $J_{1}, J_{2}, J_{3}$ and $K_{1}, K_{2}, K_{3}$

$$
\begin{aligned}
& J_{1}=\left[\begin{array}{cccc}
0 & 0 & 0 & 0 \\
0 & 0 & 0 & 0 \\
0 & 0 & 0 & -i \\
0 & 0 & i & 0
\end{array}\right], \quad J_{2}=\left[\begin{array}{cccc}
0 & 0 & 0 & 0 \\
0 & 0 & 0 & i \\
0 & 0 & 0 & 0 \\
0 & -i & 0 & 0
\end{array}\right], \quad J_{3}=\left[\begin{array}{cccc}
0 & 0 & 0 & 0 \\
0 & 0 & -i & 0 \\
0 & i & 0 & 0 \\
0 & 0 & 0 & 0
\end{array}\right] \\
& K_{1}=\left[\begin{array}{llll}
0 & i & 0 & 0 \\
i & 0 & 0 & 0 \\
0 & 0 & 0 & 0 \\
0 & 0 & 0 & 0
\end{array}\right], \quad K_{2}=\left[\begin{array}{llll}
0 & 0 & i & 0 \\
0 & 0 & 0 & 0 \\
i & 0 & 0 & 0 \\
0 & 0 & 0 & 0
\end{array}\right], \quad K_{3}=\left[\begin{array}{llll}
0 & 0 & 0 & i \\
0 & 0 & 0 & 0 \\
0 & 0 & 0 & 0 \\
i & 0 & 0 & 0
\end{array}\right]
\end{aligned}
$$

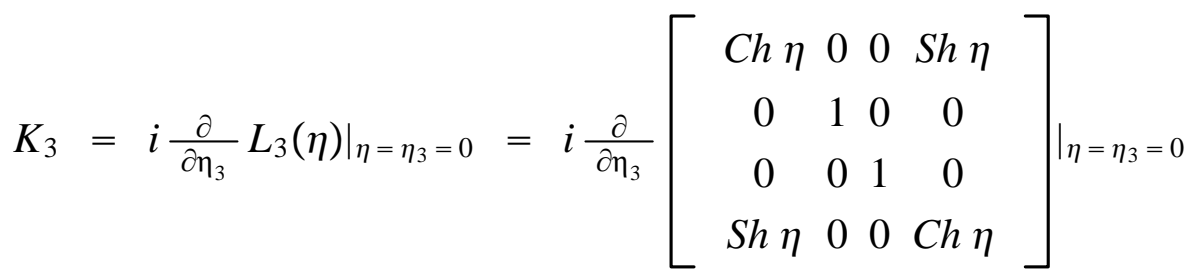

$L_{3}(\eta)$ is the familiar expression of Einstein special relativity. 
Using infinite dimensional spin augular momentum operators $\vec{\pi}_{-2,1}$ $\left(\pi_{1:-2,1}, \pi_{2:-2,1}, \pi_{3:-2,1}\right)$ of $1 \hbar$ spin boson particle, we could get six infinite dimensional matrices $\mathbb{J}_{1}, \mathbb{J}_{2}, \mathbb{J}_{3}$ and $\mathbb{K}_{1}, \mathbb{K}_{2}, \mathbb{K}_{3}$ of Lorentz Group Operators. Among them, matrix $\mathbb{K}_{3}$ is shown below

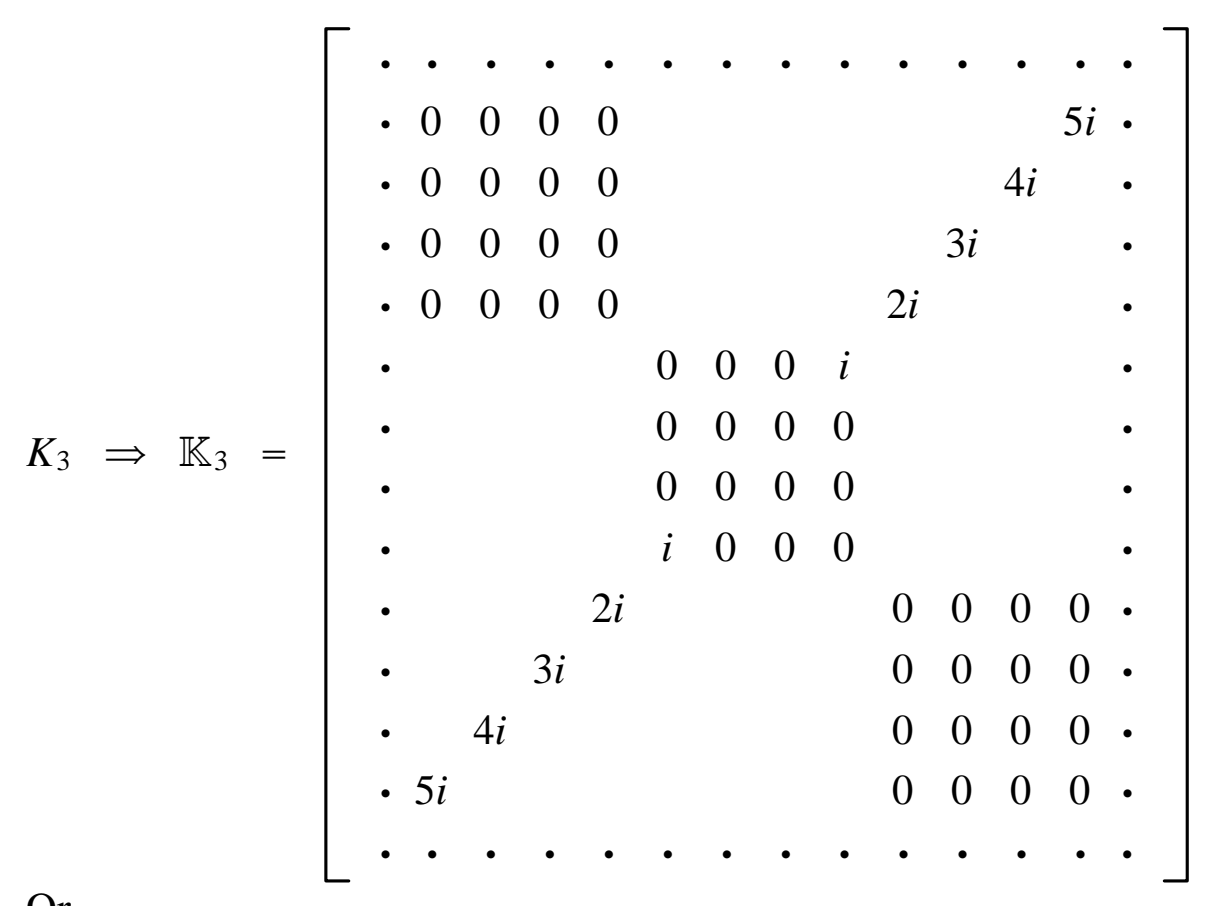

Or

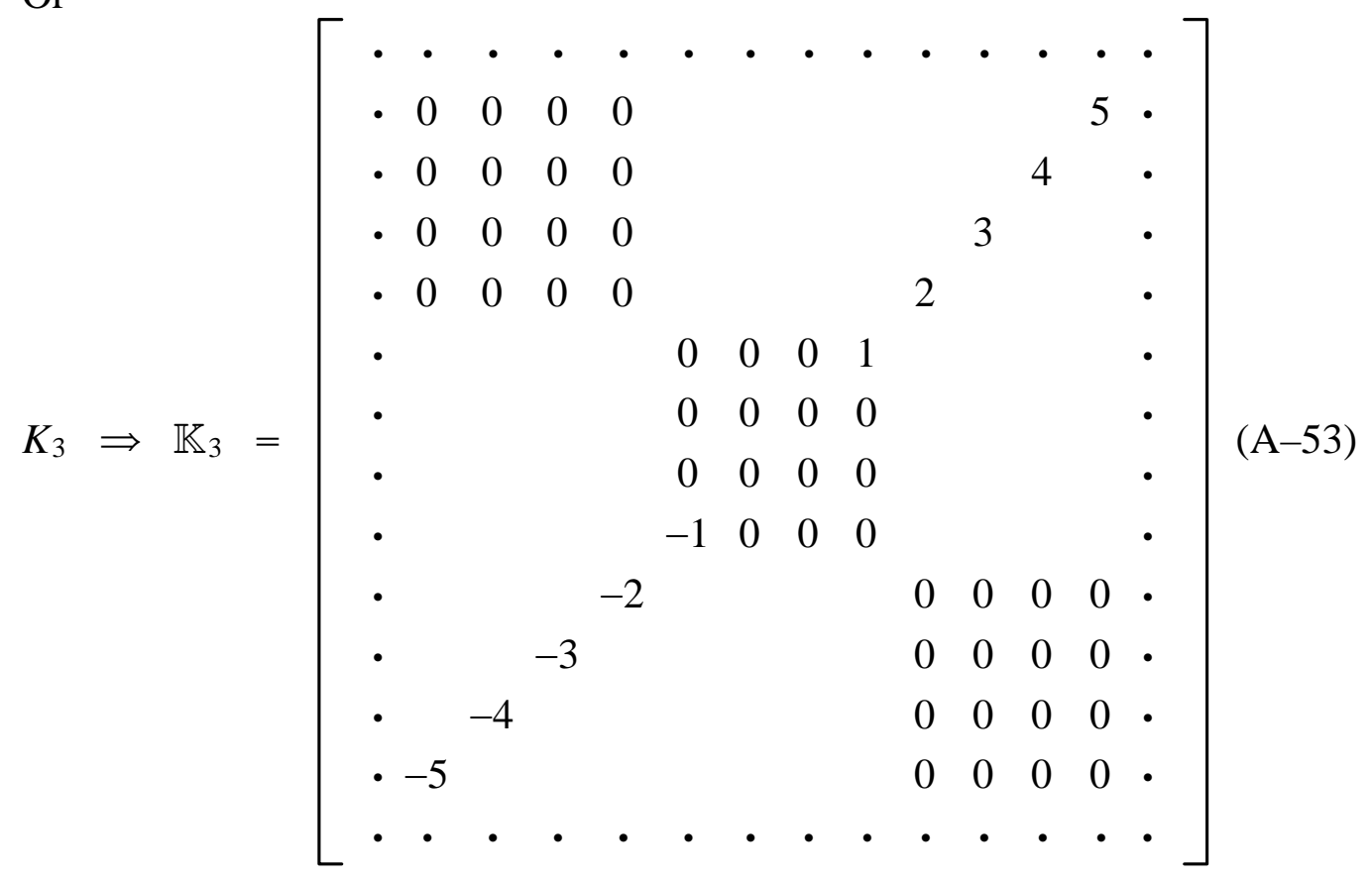

Take note of (A-52) and (A-53), they are two different types of Non-Hermitian operators, antisymmetrical matrices, base on them, proceed as follows

$$
L_{3}(\eta) \Rightarrow \mathbb{L}_{3}(\eta)
$$


From (A-57), some curious spectacles that similar to (A-48) are emerged [6] :

$$
\begin{aligned}
& \text { For }(\mathrm{A}-52) \quad \text { For }(\mathrm{A}-53) \\
& \mathbb{L}_{3}^{00}(\lambda)=\left[\begin{array}{cc}
\operatorname{Ch} \lambda & \operatorname{Sh} \lambda \\
\operatorname{Sh} \lambda & \operatorname{Ch} \lambda
\end{array}\right] \quad, \quad \mathbb{L}_{3}^{00}(\lambda)=\left[\begin{array}{cc}
\operatorname{Sech} \lambda & -\operatorname{Th} \lambda \\
\operatorname{Th} \lambda & \operatorname{Sech} \lambda
\end{array}\right] \\
& \mathbb{L}_{3}^{01}(\lambda)=\left[\begin{array}{cc}
\operatorname{Ch} 2 \lambda & \operatorname{Sh} 2 \lambda \\
\operatorname{Sh} 2 \lambda & \operatorname{Ch} 2 \lambda
\end{array}\right] \quad, \quad \mathbb{L}_{3}^{01}(\lambda)=\left[\begin{array}{cc}
\operatorname{Sech} 2 \lambda & -\operatorname{Th} 2 \lambda \\
\operatorname{Th} 2 \lambda & \operatorname{Sech} 2 \lambda
\end{array}\right] \\
& \mathbb{L}_{3}^{02}(\lambda)=\left[\begin{array}{cc}
\text { Ch } 3 \lambda & \operatorname{Sh} 3 \lambda \\
\operatorname{Sh} 3 \lambda & \operatorname{Ch} 3 \lambda
\end{array}\right], \quad \mathbb{L}_{3}^{02}(\lambda)=\left[\begin{array}{cc}
\operatorname{Sech} 3 \lambda & -\operatorname{Th} 3 \lambda \\
\operatorname{Th} 3 \lambda & \operatorname{Sech} 3 \lambda
\end{array}\right] \\
& \begin{array}{cccc}
\ldots \ldots \ldots \ldots \ldots \ldots & \ldots \ldots \ldots \ldots \ldots
\end{array}
\end{aligned}
$$

Then, Einstein Special Relativity is extended to the following so-called :

Worm Hole Special Relativity in Multi-Level Universes World: [6]

$$
\left[\begin{array}{l}
x_{0, j}^{\prime} \\
x_{3, j}^{\prime}
\end{array}\right]=\mathbb{L}_{3}^{0, j}(\eta)\left[\begin{array}{l}
x_{0, j} \\
x_{3, j}
\end{array}\right]
$$

$$
\begin{aligned}
& \text { For (A-55), have: ， For (A-56), have } \\
& x_{0}^{\prime}=\operatorname{Ch} \eta x_{0}+\operatorname{Sh} \eta x_{3} \quad, \quad x_{0}^{\prime}=\operatorname{Sech} \eta x_{0}-T h \eta x_{3} \\
& x_{3}^{\prime}=\operatorname{Sh} \eta x_{0}+\operatorname{Ch} \eta x_{3} \quad, \quad x_{3}^{\prime}=\operatorname{Th} \eta x_{0}+\operatorname{Sech} \eta x_{3} \\
& \eta=\lambda, 2 \lambda, 3 \lambda, \ldots \quad, \quad \eta=\lambda, 2 \lambda, 3 \lambda, \ldots \\
& \text { (A-58) (A-59) }
\end{aligned}
$$

and

$$
\begin{array}{llll}
\text { Sh } \eta=\beta \gamma & \text { (A-58.1) } & , & \text { Th } \eta=\beta \gamma \\
\text { Ch } \eta=\gamma & \text { (A-58.2) } & , & \text { Sech } \eta=\gamma \\
\text { Th } \eta=\beta & \text { (A-58.3) } & , & \text { Sh } \eta=\beta
\end{array}
$$

$$
\operatorname{Ch}^{2} \eta-S^{2} \eta=1(\mathrm{~A}-58.4) \quad, \quad \operatorname{Sech}^{2} \eta+T h^{2} \eta=1 \text { (A-59.4) }
$$

j) Spin Topological Space STS is the space that could discribe and help people understand how the transitions of particle spins, between various types of spin particles, are happening. Before this, the concepts of physics and math about these transitions were indistinct and blurred.

To appreciate the beauty and subtlety of STS, the FIG. below is essential. 
$\pi_{\mathrm{s}}^{2}: \hbar^{2}$

1st CSH

2nd CSH

3rd CSH

Spin: $h$

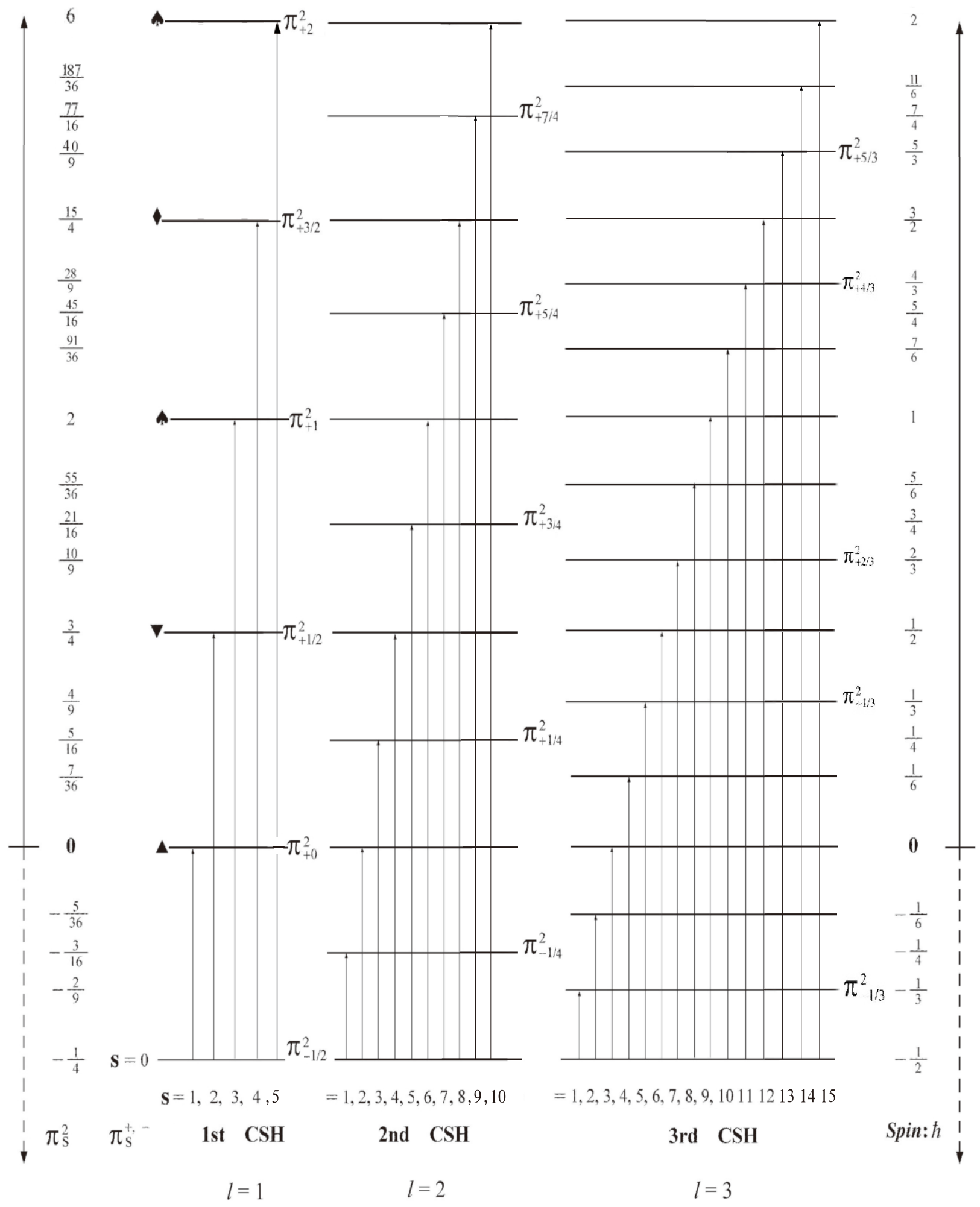

FIG. Phase transitions of Vacuum Bubble Pair $\pi_{\mathrm{s}=|i-j|}^{+,-}(l)$ from the sea floor one-fourth $-\frac{1}{4} \hbar^{2}$ deep in SAMV ocean (not to scale) in Spin topological space, $\mathbf{S T S},[4]$ 